\title{
Oxide Electronics and Vanadium Dioxide Perspective: A Review
}

\author{
A. L. Pergament, G. B. Stefanovich, and A. A. Velichko
}

\begin{abstract}
Metal-oxide-semiconductor field-effect transistors (MOSFET) have been for a long time the key elements of modern electronics industry. For the purpose of a permanent integration enhancement, the size of MOSFET has been decreasing exponentially for over decades in compliance with Moore's Law, but nowadays, owing to the intrinsic restrictions, the further scaling of MOSFET devices either encounters fundamental limits or demands for more and more sophisticated and expensive engineering solutions. Alternative approaches and device concepts are currently designed both in order to sustain an increase of the integration degree, and to improve the functionality and performance of electronic devices. Oxide electronics is one of such promising approaches which could enable and accelerate the development of information and computing technology. The behavior of $d$ electrons in transition metal oxides is responsible for the unique properties of these materials, causing strong electronelectron correlations, which play an important role in the mechanism of metal-insulator transition. The Mott transition in vanadium dioxide is specifically the phenomenon that researchers consider as a corner stone of oxide electronics, particularly, in its special direction known as a Motttransition field-effect transistor (MTFET). This review focuses on current research, latest results, urgent problems and nearterm outlook of oxide electronics with special emphasis on the state of the art and recent progress in the field of $\mathrm{VO}_{2}$-based MTFETs.
\end{abstract}

Index Terms - Oxide electronics, Mott transition FET, metal-insulator transition, transition metal oxides, vanadium dioxide.

Manuscript received November 25, 2013; accepted December 10, 2013. Date of online publication: December 15, 2013.

This work was supported by the Strategic Development Program of Petrozavodsk State University (2012 - 2016), Ministry of Education and Science of Russian Federation "Scientific and Educational Community of Innovation Russia (2009-2013)" Program through contracts no. 14.740.11.0895, no. 14.740.11.0137, no. 14.740.11.1157, no. 16.740.11. 0562, and no. 14.B37.21.0755, as well as "Development of Scientific Potential of High School" Program, contracts no. 2.3282.2011 and no. 2.2774.2011.

A. L. Pergament is with the Department of Information Measuring Systems and Physical Electronics, Faculty of Physical Engineering of Petrozavodsk State University, 185910 Petrozavodsk, Russia (e-mail: aperg@psu.karelia.ru).

G. B. Stefanovich is with the Department of Information Measuring Systems and Physical Electronics, Faculty of Physical Engineering of Petrozavodsk State University, 185910 Petrozavodsk, Russia (e-mail: gstef@yandex.ru).

A. A. Velichko is with the Department of Electronics and Electrical Power Engineering, Faculty of Physical Engineering of Petrozavodsk State University, 185910 Petrozavodsk, Russia (e-mail: velichko@ psu.karelia.ru)

\section{INTRODUCTION: HOW DID OXIDE ELECTRONICS EMERGE? FROM THE HISTORY OF THE PROBLEM}

$\mathrm{T}$ HE modern IT revolution is based on technological progress which enables an exponentially growing enhancement of the performance of electronic devices. During all the history of the development of electronic components, from a vacuum diode to modern highly integrated ICs with nanometer scale of individual elements, the question of the physical limitations on the further progress in this area arose repeatedly. After the invention of an IC by J. Kilby and R. Noyce in 1958 [1], the number of transistors on a chip roughly doubles every two years, and afterwards the processing speed and storage capacity increase correspondingly (Moore's Law). Such a dynamics is typical of all other key parameters of the ICs, the most important of which is a characteristic size of the active region $d_{\mathrm{m}}$ [2], for example, the FET effective channel length. In recent years, the issue of constraints for standard Si-based electronics has been widely discussed in the scientific literature, which is primarily associated with the possibility of further scaling toward nano-size. In this regard, in the 2007 edition of The International Technology Roadmap for Semiconductors (ITRS, http://www.itrs.net), a new section has appeared, namely "Emergent Research Device Materials", which indicates the need to develop a new generation of devices based on new physical principles [3].

Dimensional constraints of the conventional CMOS technology will not allow, apparently, overcoming the limit of $d_{\mathrm{m}}$ far beyond $10 \mathrm{~nm}$, and this can be called as a "Moore's Law violation" [4] (or, so to say, "More than Moore", - the pun which seems to originate from the ITRS authors). Note, however, that the ITRS program still optimistically claims that a theoretical limit of scaling for $\mathrm{Si}$ is not seen, and by 2026 it is planned to achieve the level of $d_{\mathrm{m}}=6 \mathrm{~nm}$ (and according to the Intel's road map $-10 \mathrm{~nm}$ by 2015 , the so called "P1274 process" [5]). Recently, a laboratory prototype of a SOI-based FET with a $3 \mathrm{~nm}$ channel length has been reported [6]. Last years, technologies with characteristic topological dimensions of 45,22 and $10 \mathrm{~nm}$ are being actively developed, and the main directions here are as follows: high- $k$ gate dielectrics, multigate structures, the use of such materials as Ge, A3B5 and graphene, Si-Ge alloys in the source and drain regions 
and strained silicon, and finally, «tri-gate» FET configuration [5] (some of these directions have also been presented in the recent review «Technology Evolution for Silicon Nanoelectronics: Postscaling Technology» [7]). Simultaneously, new technical solutions for architecture optimization (such as, e.g., multi-core processors and the Blue Gene project), system integration and innovative design are developed [4].

Alternative approaches are based on another mechanism (as compared to the field effect in Si CMOS FETs) or even on a drastic change in computational paradigm or architecture (quantum computers, neuroprocessors). Amongst the approaches utilizing new physical mechanisms, one can list, for example, the following: spintronics, superconducting electronics, single-electronics, molecular electronics, as well as one more quite recent direction, so-called "soletronics" (single atom electronics) [8]. One of such novel directions, oxide electronics, is based on the idea of application of unique properties and physical phenomena in strongly correlated transition metal oxides (TMO). Metal-insulator transition (MIT) [9] belongs to the class of the aforementioned phenomena, and many TMOs, e.g. vanadium dioxide which will be for the most part discussed in the present survey, undergo MITs as functions of temperature or electric field [10].

Complex strongly correlated TMOs, such as HTSC cuprates, CMR manganites or some interfaces (as for instance $\mathrm{LaAlO}_{3}-\mathrm{SrTiO}_{3}$ ), had first been considered as candidate materials for oxide electronics [3], [11]-[16]. The list of the devices proposed includes, for example, FETs with electron transport in complex oxide heterostructures [12], [14] (a "Sketch-FET" [13]), sensors, signal converters, memory elements, etc [3].

Afterwards, three main areas of research have emerged in the field of new functional devices of oxide electronics:

1) Elements of non-volatile memory - oxide ReRAM.

2) Devices, mainly oxide-based transistors and diodes, for transparent electronics.

3) FETs based on materials with MIT ("Mott-FET").

One cannot but admit that the above classification is rather relative. Particularly, the basic materials for transparent and flexible electronics are apparently not oxides: they are, for example, organic compounds and low-dimensional carbon materials (nanotubes, graphene) [17]-[19]. On the other hand, oxide heterostructure-based $\mathrm{p}-\mathrm{n}$ junctions as access elements (selective diodes) for ReRAM might be considered as an independent branch of oxide electronics. Also, complex perovskite oxide ferroelectrics and multiferroics, garnets for magneto-optical devices, oxide photonic crystals etc. can be utilized in various discrete oxide electronic devices [20].

Transparent electronics and oxide ReRAMs are widely discussed in the literature and described in detail in several reviews. Note, however, that the memory effect, although manifested mainly by TMOs [21]-[29], is obviously not directly associated with the electron correlation phenomena.
The most discussed models in the literature for the ReRAM mechanism in oxide structures are those based either on the growth and rupture of a metal filament inside the oxide matrix under the action of electric current, or on the redox processes responsible for the formation of some highconductivity or low-conductivity local inclusions corresponding to a particular oxygen stoichiometry. The MIT ideology is also sometimes involved to explain the properties of the structures and the memory switching mechanism therein [27]. In any case, the memory switching phenomenon seems to be associated with the ion transport [23], [24], [26], [28]. It is also appropriate to mention here the works discussing the memory effects in a material with MIT (vanadium dioxide) associated with the presence of hysteresis in the temperature dependence of conductivity [30], [31].

Typical oxides of transparent electronics ( $\mathrm{ZnO}$, ITO, In-Ga-Zn oxide, $\mathrm{Cu}_{\mathrm{x}} \mathrm{O}$, etc.) [32]-[38] do not belong to the class of TMOs, except for copper oxide, and, correspondingly, the phenomena therein are not connected specifically with the correlation effects. (Apropos, the work [38] is one of the most cited articles where the term "oxide electronics" has apparently first appeared.) Due to a sufficiently wide band gap and a large density of defect states, these oxides belong to the class of transparent conductors [39], i.e. they exhibit both a relatively high conductivity and a satisfactory transparency in the visible spectrum region. On the other hand, the developed lowtemperature synthesis methods for the thin oxide films preparation allow deposition of these films onto flexible substrates which ensures their competitive ability as compared with conventional materials of stretchable transparent electronics [40], such as organic polymers and carbon nanotubes [17]-[19].

The third of the above listed three areas of oxide electronics, i.e. that connected with transistor structures based on materials with a MIT, dates back to 1997 when the work [41] has been published in which the idea of a FET on the basis of a hypothetical molecular layer, undergoing a Mott transition, has been proposed, and in 1996 the authors of [41] had patented their idea [42]. Such a device has been called as a "Mott-FET", or MTFET Mott Transition Field Effect Transistor.

It should be noted that IBM, for example, selected three main approaches to the development of a so-called "beyond silicon" electronics [4]; they are: quantum computers, molecular electronics and a thin film MTFET. The latter is considered to be the most promising because it is closest to existing circuits and architectures [4].

Vanadium dioxide is currently considered as the most suitable material for the MTFET implementation. It should be noted that a simpler material exhibiting the Mott MIT, such as e.g. heavily doped silicon, where this transition occurs at a free charge carrier density of $n_{\mathrm{c}} \sim 3.5 \times 10^{18} \mathrm{~cm}^{-3}$ [9], would seem to be a more promising material for this purpose. However, the Mott transition in doped $\mathrm{Si}$ is the second order phase transition and hence it is not 
accompanied by a conductivity jump. On the other hand, in vanadium dioxide, the change of conductivity at the transition temperature $\left(T_{\mathrm{t}}=340 \mathrm{~K}[9]\right)$ reaches $4-5$ orders of magnitude (Fig. 1).

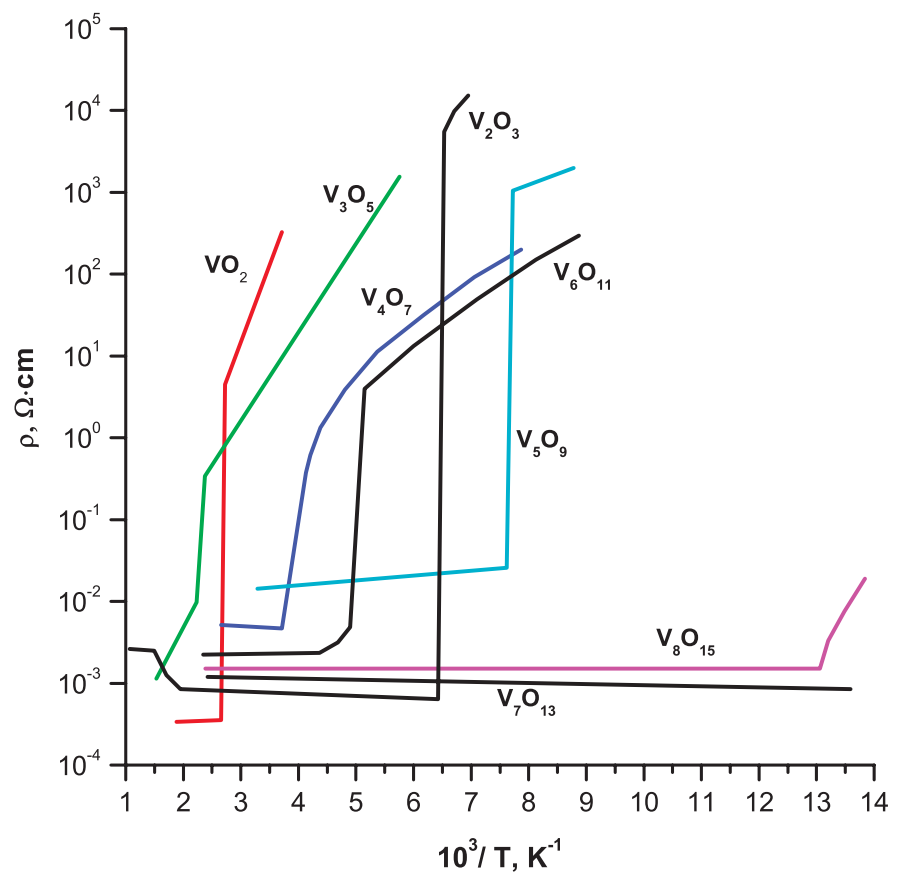

Fig. 1. Metal-insulator transitions in vanadium oxides [9], [10], [43].

In the work [41], a Mott transition field effect transistor, based on hypothetical molecular (Mott insulator) layers, in particular, such exotic materials as $\mathrm{K}^{+} \mathrm{TCNQ}^{-}$(the quasimonomer organic conductor) or $\mathrm{KC}_{60}$ (the doped fulleren) have been proposed. The version of the Mott-transitionFET based on $\mathrm{VO}_{2}$ [44] seems to be more attractive. It demonstrates high speed, low dimensions, and (what is more important) it works on the basis of the well-studied, reliable material, which has already been tested as a laboratory prototype. In addition, the important merits of vanadium dioxide are that its transition temperature is very close to room temperature and that this material is thermodynamically stable [45] as compared to other oxides in the vanadium-oxygen system (in which, by the way, there are more than ten oxides exhibiting MITs at different temperatures - see Fig. 1).

However, in order to use any compound as a material for FETs, it is necessary to be sure that the MIT therein could be induced by an electric field. This requirement has for a long time been a stumbling block to the implementation of the $\mathrm{VO}_{2}$-based MTFET. These issues are discussed in Section II of this review where we also present a brief synopsis of the vanadium dioxide physical properties, and Section III is devoted directly to the possibility of electronic control the metal-insulator transition in $\mathrm{VO}_{2}$.

Note that in contrast to the first two of the above listed directions of oxide electronics, there are almost no sufficiently detailed reviews in the literature on the topic of MTFET, except, perhaps, for the works [23] and [46]. This fact was one of the motives for writing this paper, and Section IV presents therefore the latest results and key achievements in this area. Finally, in Section V, we state the main findings and an assessment of the problems and prospects of development of oxide electronics, especially as it relates to the MTFET problem.

\section{VANADIUM DiOXIDE AS A MATERIAL FOR OXIDE ELECTRONICS}

\section{A. Introduction: Transition Metal Oxides}

Transition metal compounds (oxides, chalcogenides, etc.) are of considerable interest for basic research in condensed matter physics and chemistry and, on the other hand, these materials are promising from the viewpoint of the use in various fields of engineering due to their diverse, and often unique, properties [47]-[50]. A set of valence states, associated with the existence of unfilled $d$-shells in the atoms of transition metals, leads to formation of several oxide phases with different properties, ranging from metallic to insulating. Moreover, lower oxides of, e.g., Ti, $\mathrm{W}$, Mo, and $\mathrm{Nb}$ not only exhibit metallic properties, but under proper doping may be even superconductors [49]. It is exactly a reversible formation of conductive filaments of lower oxides that in some cases accounts for the nonvolatile memory mechanism in TMO-based structures [28], [29]. One of the expressive properties of transition metal compounds are electronic instabilities, such as metalinsulator phase transitions [9], [47], [48] and effects of electrical switching [51], [52]. In other words, TMOs exhibit an astonishing array of functionalities that result from a combination of the strongly polarizable metaloxygen bond and strong correlations [50]. It is specifically the behavior of $d$-electrons in the compounds of transition metals that is responsible for the unique properties of these materials, causing strong electron-electron correlations, which play an important role in the mechanisms of such phenomena as, e.g., MITs, high- $T_{\mathrm{c}}$ superconductivity (HTSC), and colossal magnetoresistance (CMR), which are inherent to many TMOs [53]. The point is that the wave functions of $d$-electrons form narrow bands, and the behavior of electrons in these bands is characterized by strong electron-electron correlations, that is, in compounds of $d$-elements, a characteristic energy of electron interactions is comparable with the band width, i.e. with the kinetic energy of an electron. The examples of such electron-correlation effects are the above mentioned phenomena of HTSC and CMR. Also, in many cases, these electron-correlation effects bring about the MIT, and one of the well-known examples is the Mott-Hubbard transition [9].

The vanadium-oxygen system may serve as an excellent illustration of the above-said about the unique properties of TMOs. Vanadium oxide Magneli phases, forming the homologous series $\mathrm{V}_{\mathrm{n}} \mathrm{O}_{2 \mathrm{n}-1}(3 \leq \mathrm{n} \leq 9)$, may be considered as intermediate structures between the end members $\mathrm{VO}_{2}$ $(\mathrm{n} \rightarrow \infty)$ and $\mathrm{V}_{2} \mathrm{O}_{3}(\mathrm{n}=2)$. With one exception $\left(\mathrm{V}_{7} \mathrm{O}_{13}\right)$, the 
vanadium Magneli phases exhibit MITs [47] as functions of temperature-see Fig. 1 and Table I. Vanadium pentoxide is an insulator with $E_{\mathrm{g}}=2.5 \mathrm{eV}$ [49], and lower vanadium oxides $\mathrm{VO}_{\mathrm{x}}(x<1)$ exhibit metallic properties. Vanadium monoxide $\mathrm{VO}_{1+\mathrm{y}}(-0.15<y<0.25)$, with a change of the parameter $y$, undergoes a transition from a metallic state to a Fermi liquid state with the Anderson localization [55].

TABLE I

VANADIUM OXIDES [47], [48], [54]

\begin{tabular}{ccc}
\hline \hline$n$ in formula $\mathrm{V}_{\mathrm{n}} \mathrm{O}_{2 \mathrm{n}-1}$ & Oxide & $T_{\mathrm{t}}, \mathrm{K}$ \\
\hline$(1)$ & $\mathrm{VO}$ & - \\
$(2)$ & $\mathrm{V}_{2} \mathrm{O}_{3}$ & 150 \\
3 & $\mathrm{~V}_{3} \mathrm{O}_{5}$ & 450 \\
4 & $\mathrm{~V}_{4} \mathrm{O}_{7}$ & 240 \\
5 & $\mathrm{~V}_{5} \mathrm{O}_{9}$ & 130 \\
6 & $\mathrm{~V}_{6} \mathrm{O}_{11}$ & 170 \\
7 & $\mathrm{~V}_{7} \mathrm{O}_{13}$ & - \\
8 & $\mathrm{~V}_{8} \mathrm{O}_{15}$ & 70 \\
$(\infty)$ & $\mathrm{VO}_{2}$ & 340 \\
$\left.(-6)^{*}\right)$ & $\mathrm{V}_{6} \mathrm{O}_{13}$ & 150 \\
$(-2)$ & $\mathrm{V}_{2} \mathrm{O}_{5}$ & - \\
\hline \hline
\end{tabular}

${ }^{*} \mathrm{~V}_{6} \mathrm{O}_{13}$ and $\mathrm{V}_{2} \mathrm{O}_{5}$ formally correspond to the series $\mathrm{V}_{\mathrm{n}} \mathrm{O}_{2 \mathrm{n}-1}$ with negative $n$, though actually they belong to the Wadsley phases $\mathrm{V}_{2 \mathrm{n}} \mathrm{O}_{5 \mathrm{n}-2}[56]$.

\section{B. Properties of $\mathrm{VO}_{2}$}

Vanadium dioxide has been described in detail in a number of reviews and monographs [9], [47], [48], [55], mainly in light of the MIT in this compound. In stoichiometric single crystals, the transition occurs at $T=340 \mathrm{~K}$ and the resistivity change reaches a factor of $10^{5}$ over a temperature range of $0.1 \mathrm{~K}$. This MIT is a first order phase transition with the latent heat $q=4.27 \mathrm{~kJ} / \mathrm{mol}$. Hysteresis associated with this transition is of about $2 \mathrm{~K}$. The magnitude of this transition and the narrowness of the hysteresis loop is a good indication of how close the stoichiometry is to $\mathrm{VO}_{2}$. Small stoichiometric deviations destroy the sharpness of the transition and increase the hysteresis width. The crystalline state of the material also has an influence; polycrystalline material will have a broader transition than single crystals. In addition, the transition temperature also depends on the crystalline state, oxygen non-stoichiometry, and doping. The temperature dependence of resistivity is shown in Fig. 2.

At high temperature, vanadium dioxide has a rutile-type structure (Fig. 3) with vanadium atoms equally spaced along the corresponding $c_{r}$-axis (Fig. 3, b, c). At the transition to the low-temperature state these vanadium atoms pair along the $c_{r}$-axis with a slight twist which leads to a monoclinic symmetry (Fig. 3, a) [47].

The conductivity activation energy of the $\mathrm{VO}_{2}$ semiconductor phase immediately below the transition temperature is $E_{\mathrm{a}} \approx 0.5 \mathrm{eV}$, which is consistent with the band gap found from optical measurements $E_{\mathrm{g}} \sim 1 \mathrm{eV}$, i.e. $E_{\mathrm{a}}=0.5 E_{\mathrm{g}}$, like in an intrinsic semiconductor. However, the conductivity is of n-type (e.g., from Hall measurements),

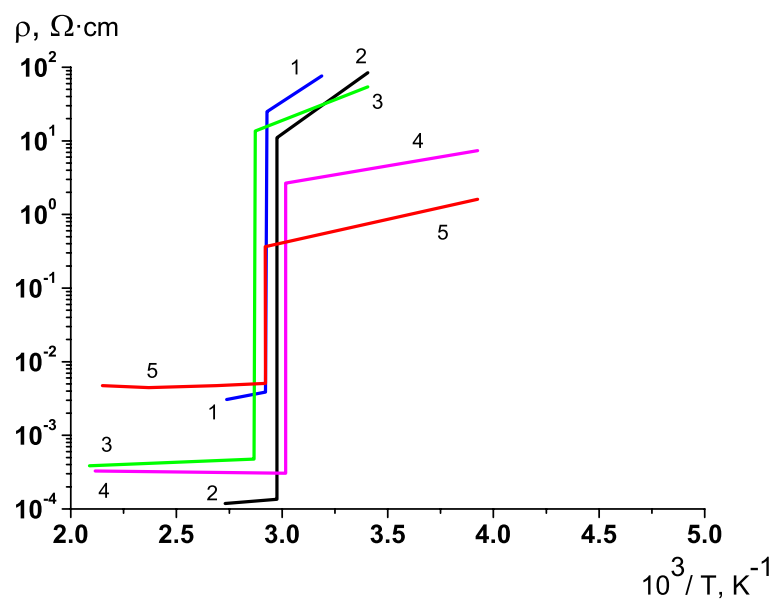

Fig. 2. Resistivity of $\mathrm{VO}_{2}$ single crystals along the $c$ axis as a function reciprocal temperature ( 1 - 5: data from different works; for references see [9], 1st edition of 1974).

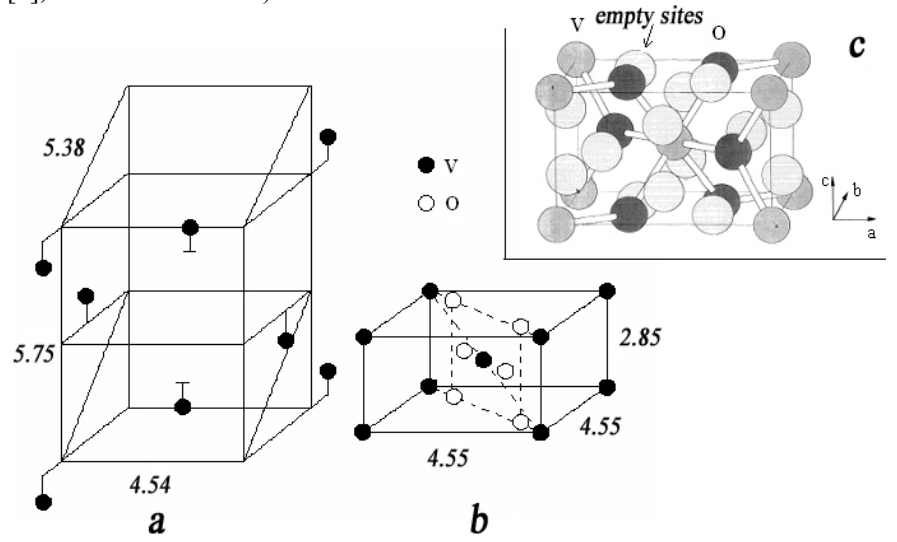

Fig. 3. Crystal structure of semiconductor (a) and metal (b) phases of $\mathrm{VO}_{2}$. Distances in angstroms are indicated. Part (a) shows only displacements of the vanadium atoms (schematically). Unit cell of tetragonal phase is shown in inset (c) [10], [47].

and such a discrepancy is quite typical for oxides, especially, for TMOs [49], [50]. The mobility of charge carriers is $0.5-1 \mathrm{~cm}^{2} / \mathrm{V} \cdot \mathrm{s}$ [9] and it does not change at the transition, therefore the whole of conductivity jump is due to the change in charge carrier density. The pointed out mobility value, as well as the value of $\rho_{\mathrm{s}} \approx 1-10 \Omega \cdot \mathrm{cm}$ (see Fig. 2), yield the charge carrier density in the semiconducting phase (close to the transition onset and at room temperature) $n_{\mathrm{s}} \sim 10^{18}-10^{19} \mathrm{~cm}^{-3}$.

The available data on the effect of doping and non-stoichiometry on the properties of $\mathrm{VO}_{2}$ [9], [47] can be summarized as follows. The oxygen deficiency, as well as the presence of impurities of the $\mathrm{Nb}(5+)$ group, lowers both $T_{\mathrm{t}}$ and the activation energy $E_{\mathrm{a}}$. It is specifically the presence of impurities or oxygen non-stoichiometry that results in a wide spread of the values of $T_{\mathrm{t}}, E_{\mathrm{a}}$ and $\rho_{\mathrm{s}}$ (see Figures 2 and 4). Moreover, in some cases, alloying may result in complete suppression of the transition and metal conductivity appears at temperatures $<300 \mathrm{~K}$ (for example, under tungsten doping to a level of about 9.5 at. \% of $\mathrm{W}$ [57], [58]; the doping effect will be also discussed below in Section II.C). The oxygen excess, or doping with impurities 
of the $\mathrm{Fe}(3+)$ group, usually results in an increase in the value of $T_{\mathrm{t}}$, and the data on $E_{\mathrm{a}}$ and $\rho_{\mathrm{s}}$ are contradictory. However, at any changes in the composition or structure there is some empirical correlation between the resistance of the semiconductor phase and the activation energy: as the $\rho_{\mathrm{s}}=\left(\sigma_{\mathrm{s}}\right)^{-1}$ increases, the $E_{\mathrm{a}}$ increases too in complete accordance with the Mott MIT theory and the concept of a minimum metallic conductivity [55]. This correlation is demonstrated by Fig. 4.

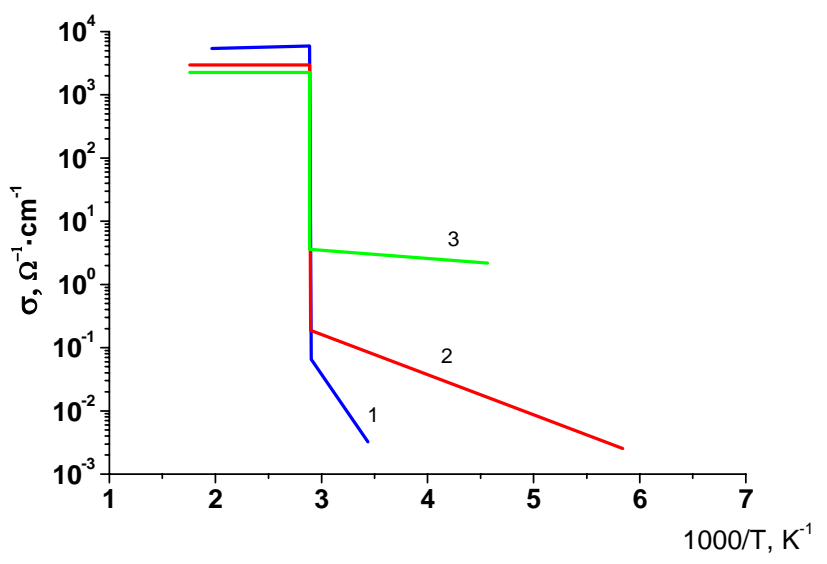

Fig. 4. Temperature dependences of conductivity of three samples of $\mathrm{VO}_{2}$ with different oxygen stoichiometry [59]. One can see that as the $\rho_{\mathrm{s}}$ increases (from sample 3 to sample 1), the slope, i.e. the value of $E_{\mathrm{a}}$, increases too.

The MIT mechanism in $\mathrm{VO}_{2}$ has for a long time been a matter of especial concern and a subject of discussion [9], [47], [60]. Note that, as was mentioned in Section I above, this issue is of fundamental importance from the viewpoint of feasibility of a $\mathrm{VO}_{2}$-based MTFET. It would seem that changes in the crystal structure at the transition (doubling of the unit cell period) can be treated in terms of the Peierls transition [61]. In addition, a number of direct experiments, for example, the pressure dependence of $T_{\mathrm{t}}$ [59] suggest, according to the authors of [47], the important role of phonons in the MIT mechanism in vanadium dioxide. On the other hand, the effects associated with electronic correlations are observed both in the semiconducting and in metallic phases of vanadium dioxide [48], [62]. Experiments on the initiation of the MIT using photogeneration of charge carriers by femtosecond laser pulses [63], [64] without lattice heating to $T=T_{\mathrm{t}}$ indicate that the electron-electron interaction is also of importance for a correct description of the transition. This is supported by studies of the MIT in thin films of amorphous vanadium dioxide [62] obtained by anodic oxidation [45], [65].

It has been shown [62], [65] that the XRD spectrum of the anodic vanadium oxide exhibits diffuse maxima typical for amorphous materials. The occurrence of the MIT in these structurally disordered $\mathrm{VO}_{2}$ films has been indicated by an abrupt and considerable (although reversible) change in the conductivity at $T=310-330 \mathrm{~K}$, and this temperature practically coincides with the transition temperature for the
MIT in crystalline $\mathrm{VO}_{2}, T_{\mathrm{t}}=340 \mathrm{~K}$. The importance of these results consists in the evidence that the metal-insulator phase transition in $\mathrm{VO}_{2}$ is preserved in the absence of a long-range crystallographic order. This, in turn, suggests that electron-electron correlations play an important role in the transition mechanism, and hence the MIT in $\mathrm{VO}_{2}$ is an electronic Mott transition, rather than a structural Peierls transition for which, in the first approximation, a long-range crystallographic order is necessary.

We thus arrive at a conclusion that the experimental facts presented above could not be described only in terms of a purely phonon mechanism of the MIT in $\mathrm{VO}_{2}$, and a correct description of this transition should involve the Mott-Hubbard electron-correlation effects [44], [57], [62], [66], [67].

It has been shown in the work [67] that, on the basis of the Mott criterion for MIT [9]

$$
a_{\mathrm{H}} n_{\mathrm{c}}^{1 / 3} \approx 0.25,
$$

an expression for the correlation length $\xi$, identical to that for the coherence length in the theory of superconductivity, can be obtained:

$$
\xi=2 \hbar v_{\mathrm{F}} / \pi \Delta .
$$

Here $n_{\mathrm{c}}$ is the critical charge carrier density for MIT, $\hbar-$ the Planck constant, $v_{\mathrm{F}}-$ the electron Fermi velocity, and $\Delta$ is the correlation energy gap. The Bohr radius in (1) is

$$
a_{\mathrm{H}}=\varepsilon \hbar^{2} / m^{*} e^{2},
$$

where $\varepsilon, m^{*}$ and $e$ are, respectively, the material dielectric constant, charge carrier effective mass, and charge of an electron. This correlation length (2) characterizes the size of an electron-hole pair (in an excitonic insulator) or the effective Bohr radius $a_{\mathrm{H}}$ (as, e.g., in doped semiconductors). The relation obtained is used for calculation of the correlation length in vanadium dioxide, and the presence of two characteristic lengths $\left(\xi_{1} \sim 20 \AA\right.$ and $\left.\xi_{2} \sim 1-2 \AA\right)$ is found. This is associated with the specific features of the transition mechanism in $\mathrm{VO}_{2}$ : this mechanism represents a combination of the purely electronic Mott-Hubbard contribution and the structural (Peierls-like) one. It is shown, however, that the driving force of the MIT in $\mathrm{VO}_{2}$ is the electronic Mott-Hubbard transition. The electron-correlation contribution to the energy gap, corresponding to this transition, is $\Delta_{1} \sim k T_{\mathrm{t}} \sim$ $0.1 \mathrm{eV}$ and $\xi=\xi_{1}=a_{\mathrm{H}}$. The modulation of the electronic spectrum results then in distortion of the crystal structure accompanied by formation of the gap $\Delta_{2} \sim E_{\mathrm{g}}$ and by the complete localization of electrons onto vanadium atoms with the localization radius $R=\xi_{2}$.

Note however that such a strict classification of the 
transition as either a purely Peierls or a purely MottHubbard is rather schematic and oversimplified. In fact, the MIT in vanadium dioxide is a two-stage (i.e. occurring through an intermediate phase with $\Delta=\Delta_{1}$ and $\left.\xi=\xi_{1}\right)$, and the Mott transition should be considered as merely a trigger mechanism which then initiates the structural transformation.

We again emphasize that the conclusion made is based on experimental evidence, and the above arguments (concerning $\xi, \Delta, a_{\mathrm{H}}, n_{\mathrm{c}}$ etc) represent only a model designed to remove the contradictions and disputes about the Mott or Peierls nature of the MIT in $\mathrm{VO}_{2}$. Experimental facts in question indicate that the transition might be initiated by an increase in the free charge carrier density (without heating and without affecting the lattice, i.e. not under, for example, doping or pressure) under photo-generation [63], [64], injection [44], or high-field generation at switching [68].

Generally, the question about the MIT mechanism in $\mathrm{VO}_{2}$ (posed as "either Mott, or Peierls transition" [60]) has no sense because, in fact, this mechanism is essentially dual [67] and can be treated as a "Mott-Peierls transition" [69], [70].

Nonetheless, one should be aware of the fact (and take this fact into account when analyzing the $\mathrm{VO}_{2}$-based electronic devices) that the initiating mechanism is still the Mott transition [44], [57], [62], [67], [68]. It is fair to admit that some researchers continue to insist that the Peierls instability plays a dominant role in the transition mechanism [61]. Even the discoverers of the effect of femtosecond laser excitation of MIT in $\mathrm{VO}_{2}$, on the basis of their experiments, deem the photogeneration of nonequilibrium electrons leads primarily to structural changes, and only these changes are in turn responsible for the electronic transition [64]. Other researchers formulate this conclusion in the opposite way, speaking of a correlation-assisted Peierls transition [71]. We believe that even if consider the Peierls structural instabilities and electronic Mott-Hubbard correlations in $\mathrm{VO}_{2}$ to be equal in their contribution to the MIT mechanism, it should still be recognized that the latter are, figuratively speaking, a little bit "more equal than others". On the whole, the problem is akin to the famous philosophical dilemma "which came first, the chicken or the egg?" and has about the same practical benefits from the ongoing debates in this field.

\section{Transition Mechanism: The Role of Electron Correlations}

In this section we would like to acquaint a reader with some recent experimental evidences confirming the important role of electron correlation effects in the MIT dynamics in $\mathrm{VO}_{2}$ [72]-[82]. Particularly, the authors of [72] have studied the electrically triggered MIT in $\mathrm{VO}_{2}$ thin films at temperatures far below the structural phase transition temperature $340 \mathrm{~K}$. It is shown that the observed weak temperature dependence of the threshold voltages is smaller than that predicted for a purely current induced Joule heating effect and may include contribution from field effect or carrier injection under applied field, and a linear relationship between $\log (I / V)$ and $(V)^{1 / 2}$ indicates the Poole-Frenkel conduction mechanism [72], [82]. These findings confirm our previous suggestions [68] concerning the switching and MIT mechanisms, as well as the PooleFrenkel charge transfer mechanism, in vanadium dioxide.

Next, it is shown in the work [73] that voltage controlled oscillations occurring in micro-beams of vanadium dioxide are a result of the reversible insulator to metal phase transition. Modeling of this phenomenon shows that, "while temperature plays a critical role in the vanadium dioxide phase transition, electrically induced heating cannot act as the primary instigator of the oscillations", and electric field, on the contrary, is "the most likely candidate for driving the phase transition" [73]. The study of local probe currentvoltage (STM) and electrostatic force-voltage (AFM) characteristics of electric-field-induced insulator to metal transition in $\mathrm{VO}_{2}$ thin film [79] also shows that electric field alone (i.e. without a heating up to the equilibrium transition temperature) is sufficient to induce the MIT. The authors of [78] have measured independently the MIT and structural phase transition hysteresis in epitaxial $\mathrm{VO}_{2}$ films, and shown that they are not congruent. These results are also in accordance with the data of [74] concerning an intervening phase of correlated metal (Fig. 5), between metal and insulator states, which seems to be identical to the intermediate phase with $\Delta=\Delta_{1}$ и $\xi=\xi_{1}$ in terms of the work [67].

The review of the properties of $\mathrm{VO}_{2}$ presented in the previous section shows that important information about the properties of this compound can be obtained from the study of the effect of doping on the MIT characteristics such as $T_{\mathrm{t}}$, the temperature transition width and the hysteresis width, the values of electrical conductivity in the semiconductor and metal phases, as well as the relationships between them.

Since the doping with tungsten, as was noted above, lowers $T_{\mathrm{t}}$ (by about 10 to $30 \mathrm{~K}$ per at. \% of $\mathrm{W}$ [57], [58], [80], [81]), this can be treated in terms of the Mott MIT. Substitution of a $\mathrm{W}^{6+}$ ion for a $\mathrm{V}^{4+}$ ion produces two $\mathrm{V}^{3+}$ ions, as dictated by the electroneutrality condition. The formation of donor defects leads to a decrease in resistivity and reduction in the MIT temperature because of the decrease in the band gap width [57], [80], and the reduction of $T_{\mathrm{t}}$ upon doping lends support to the electronic Mott mechanism of the phase transition in vanadium dioxide.

A different mechanism of the tungsten impurity influence, based predominantly on structural processes, has been proposed in [81] where the behavior of an isolated $\mathrm{W}$ dopant and its influence on the $\mathrm{VO}_{2}$ host lattice has been investigated by X-ray absorption spectroscopy and the first-principle calculations. The authors assert that, in their work, a clear atomic structure of $\mathrm{W}$ atoms in $\mathrm{VO}_{2}$ has been unveiled, which possesses an intrinsically symmetric 


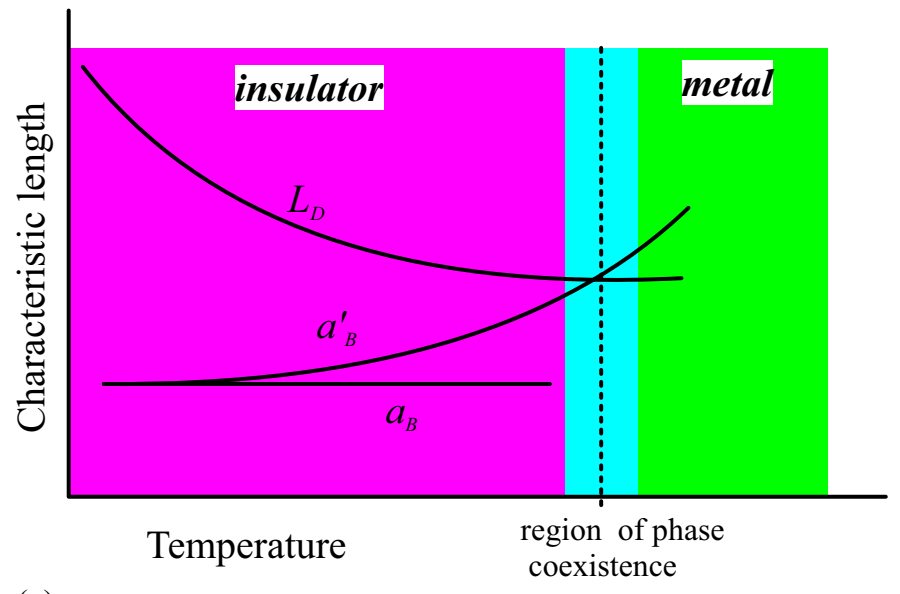

(a)

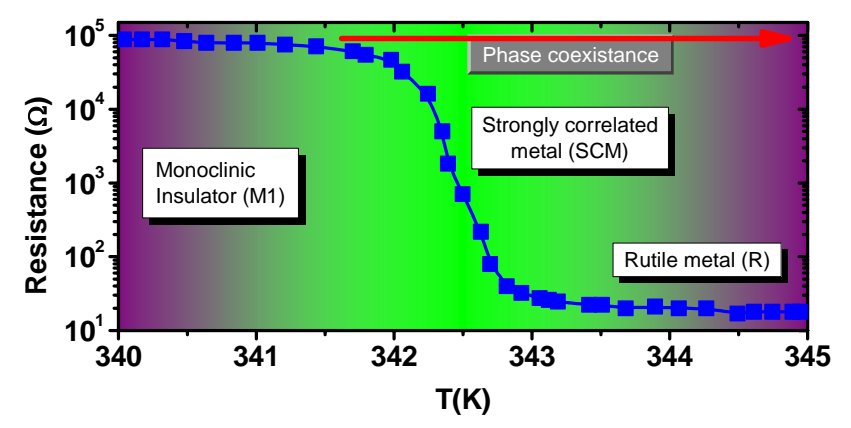

(b)

Fig. 5. Intermediate state in vanadium dioxide (a) [43] treated as a strongly correlated metal (b) [74].

tetragonal structure, removing the twisting (see Fig. 3, a) of the nearby asymmetric monoclinic $\mathrm{VO}_{2}$ lattice and driving it thereby towards the rutile phase. However, such a purely structural approach [81] does not explain the experimentally observed reentrant transition to the semiconducting state at a doping level of more than 10 at. $\%$ of W (see Fig. 6) or, as suggested in [58], to the Mott insulator state.

It should be noted that a huge number of works on doping (especially, by tungsten) amongst all the publications on $\mathrm{VO}_{2}$ is explained by the importance of these studies from the viewpoint of possible technical applications (which will be in rather more detail discussed in Section V below), because doping allows the control the transition temperature and other MIT parameters, in particular, the shift of $T_{\mathrm{t}}$ toward room temperature - see, for example, [57], [80], [83], [84].

Hydrogen insertion, likewise the above-described $\mathrm{W}$-alloying, to a certain level of the hydrogen concentration $\mathrm{x} \sim 0.3$ in $\mathrm{H}_{\mathrm{x}} \mathrm{VO}_{2}$, also leads to metallization of vanadium dioxide [75]-[77], although the mechanism is obviously different, and there is still no consensus concerning the interpretation of this phenomenon. Particularly, it is argued in [76] that hydration initiates the electronic transition to the metallic phase which is not accompanied by a change in the structure from the monoclinic to tetragonal symmetry, while in [75] the hydration-induced metallization is supposed to be associated with the distortion of the structure caused by the hydrogen atom insertion. Recently, it has been shown that the MIT in $\mathrm{VO}_{2}$ nano-beams can be modified by doping with atomic hydrogen using the catalytic spillover method [77]. This process is completely reversible, and the transition vanishes when the doping level exceeds a threshold value. Raman and conventional optical microscopy, electron diffraction and transmission electron microscopy provide evidence that the structure of the metallic post-hydrogenation state is similar to that of the rutile state. First-principles electronic structure calculations confirm that this hydrogenated rutile structure is energetically favored, and such doping favors metallic behavior of vanadium dioxide from both the Mott and Peierls perspectives [77].

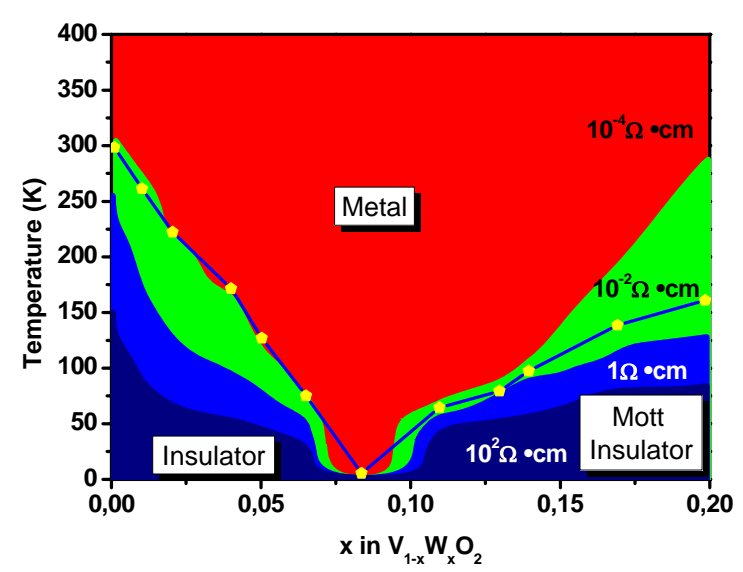

Fig. 6. Phase diagram restored from the data of [58].

Thus, introduction of donor-type defects $\left(\mathrm{Nb}^{5+}\right.$ or $\mathrm{W}^{6+}$ impurities, inserted $\mathrm{H}^{+}$ions, oxygen vacancies) results in a lowering of $T_{\mathrm{t}}$ in vanadium dioxide. Also, the MIT temperature has been found to depend on the free-carrier density determined by the stoichiometry defect concentration [85].

As was already said, important information on the mechanism of MIT might be, in principle, obtained from the study of light-induced transition [63], [64]. We farther discuss some more recent works in this field [86]-[91] where the main conclusions of the earlier work [64] are confirmed, and some new results are also reported.

Particularly, the photoinduced insulator-metal transition in $\mathrm{VO}_{2}$ has been studied by means of broadband timeresolved reflection spectroscopy [89] which allows the authors to separate the response of the lattice vibrations from the electronic dynamics and observe their individual evolution. It is shown that an ultrafast change in the lattice potential drives the structural transition and that the electronic properties of the material evolve on a different, slower time scale. This separation of time scales suggests that the early state of $\mathrm{VO}_{2}$, immediately after photoexcitation, is a nonequilibrium state that is not well 
defined by either the insulating or the metallic phase [89]. A similar transient state with a characteristic time scale of about $10 \mathrm{ps}$ has been reported in [86], and this state seems to be related to the SCM state (see Fig. 5) discussed in [74]. Also, a general mechanism for photoinduced phase transitions has been developed [88] and demonstrated to be applicable for the MIT of $\mathrm{VO}_{2}$ using a $20 \mathrm{~nm}$ Au injection layer. It is shown that the $\mathrm{Au}$ film is the source of nonequilibrium electrons driving the phase transition. Photoinjection of hot electrons from a metal $\mathrm{Au}$ contact resulting in a MIT in accordance with this mechanism has been experimentally studied in the work [90]. Photoassisted bistable switching in planar junction devices based on $\mathrm{VO}_{2}$ thin films has been investigated in [87], and the results are interpreted in terms of the photoinduced electronic Mott MIT which is not accompanied by a structural transformation.

Also, the dynamics of the reverse process, namely, the transition from metallic to insulating state has been studied in recent work [91], and it has been shown that the optical excitation and fast recovery of ultrathin $\mathrm{VO}_{2}$ films provides a practical method to reversibly switch between the monoclinic insulating and tetragonal metallic state at nanosecond time scales.

The above presented data, in our opinion, clearly evidence that the electronically controllable MIT in vanadium dioxide is actually feasible. However, in order to use $\mathrm{VO}_{2}$ as the basic material for an MTFET, the sole fact of electronic control is not sufficient, but such parameters as the operating speed and the ability of transition to nanosize are also of importance. Note that the fundamental limit of the switching time from the semiconductor to the metallic state, associated with the mechanism of the MIT in $\mathrm{VO}_{2}$ and determined from experiments on the photoinitiation of transition, is of about $100 \mathrm{fs}$ [64]. However, the switching time in electric schemes will naturally be higher. As for the scalability, it is also absolutely not obvious a priori that the transition preserves in nano-scale and is not suppressed due to the size effects on the MIT.

The delay time $t_{\mathrm{d}}$ in the $\mathrm{VO}_{2}$-based structures has been investigated in many studies. In the work [92], it is determined as $4.5 \mathrm{~ns}$ for the interelectrode gap of $d=125 \mathrm{~nm}$ in a planar switch. Theoretical estimates, which are consistent with the experimental data, yield $t_{\mathrm{d}} \approx 1 \mathrm{~ns}$ for $d=20 \mathrm{~nm}$ [93]. Note that in the sandwich structures with a film thickness of $d=100 \mathrm{~nm}$, the delay time can reach a value of $\sim 0.1 \mathrm{~ns}$ [44]. As the thickness increases, the switching time rises parabolically because of a higher time constant $R C$ [94] - see Fig. 7.

The review [46] summarizes literature data on measurements of the characteristic time for the MIT development in $\mathrm{VO}_{2}$ (Table 2 in [46]); it is shown that in different experiments on initiating the transition via microwave or optical pumping, this time lies within the femto- to picosecond range, and for electrical switching the

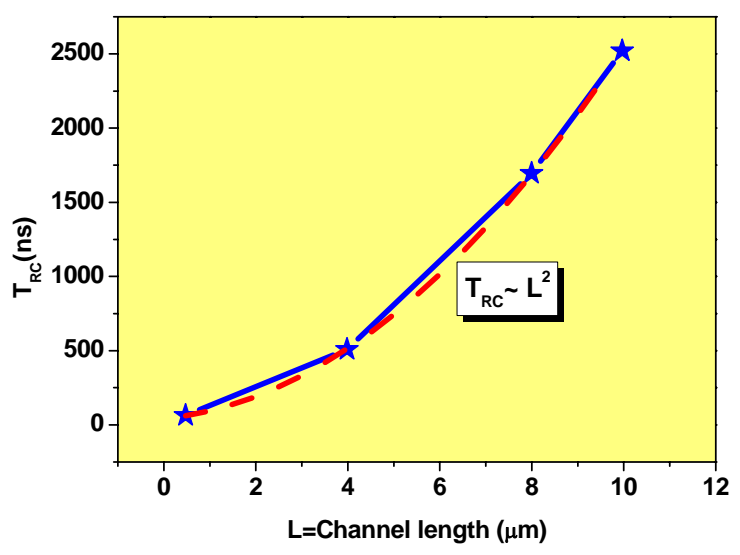

Fig. 7. Switching time as a function of channel length [94].

ultimate value of $t_{\mathrm{d}}$ is $9 \mathrm{~ns}$. We also emphasize that almost all the papers mentioned above treat the results on the $t_{\mathrm{d}}$ measurements in terms of the electron-induced Mott transition.

Thus, in order to obtain the minimum switching time and hence the maximum device operation speed, one needs, obviously, to diminish the device dimensions. Plenty of works in the field of nanostructured $\mathrm{VO}_{2}$ in recent years makes it impossible even to quote them all here. Therefore, we confine ourselves only to the most general observations and a few selected references. In particular, the works [95], [96] provide an overview of the properties of nanostructured TMOs (HTSC, CMR-manganite, $\mathrm{Fe}_{3} \mathrm{O}_{4}$ and $\mathrm{VO}_{2}$ ); see also [97], where the dimensional effects on the MIT in $\mathrm{VO}_{2}$ are studied, and references therein to the most significant works on the MIT in ultrathin films, nano-wires and other $\mathrm{VO}_{2}$-based nano-objects, published prior to 2010 .

It is discussed in the work [97] that vanadium dioxide has various potential applications in electronics due to the metal-insulator MIT, and on the other hand it is known that oxide structures with nanometric dimensions exhibit properties different from bulk oxide materials because of the spatial confinement and the proximity of the substrate. However, in order to integrate $\mathrm{VO}_{2}$ into the thriving nanoscale electronics, it is necessary to explore the MIT in this material in thin film nano-structures. It has been shown [97] that there is a fundamental dimensional restriction for the transition to occur even for pure epitaxial $\mathrm{VO}_{2}$ nano-films and nano-wires. This is associated with the fact that any phase transition turns out to be impossible when the system size is decreased below a certain characteristic length $d_{\mathrm{c}}$. This dimension is estimated to be $d_{\mathrm{c}} \sim \xi$ (where $\xi$ is the correlation length, $\sim 2 \mathrm{~nm}$ for $\mathrm{VO}_{2}$ [67]), and, on the basis of the available experimental data, it is shown that the transition temperature falls as the characteristic size (film thickness or nano-wire radius) diminishes (Fig. 8), though the predicted theoretical limit of $2 \mathrm{~nm}$ is not still experimentally achieved by now. The main conclusion one can arrive at from these works consists therefore in the fact that the MIT in $\mathrm{VO}_{2}$ preserves in nanoscale down to at least 2 to $10 \mathrm{~nm}$. 


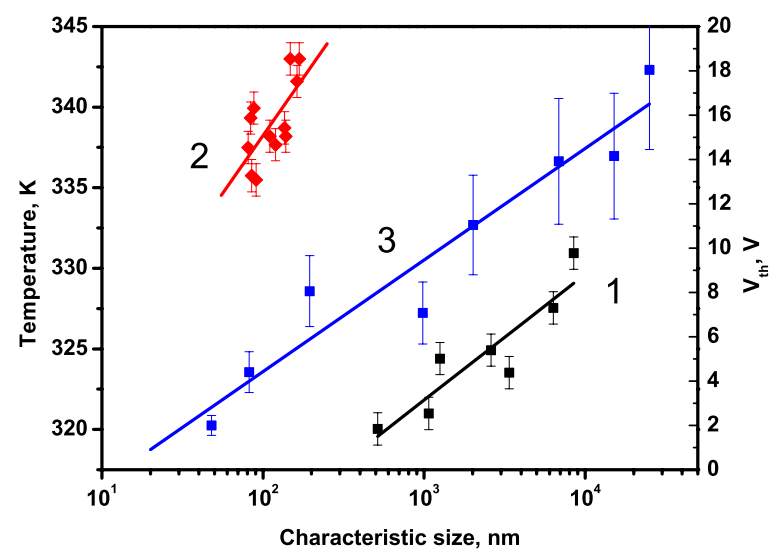

Fig. 8. Transition temperature of vanadium dioxide as a function of the nanowire width (1) and film thickness (2), and threshold voltage as a function of the film thickness (3) for the switching structures metal- $\mathrm{VO}_{2}-$ metal (see [97] and references therein).

Concluding this brief discussion of the MIT in nanostructured $\mathrm{VO}_{2}$, we mention also the work [98] where a new high-yield method of doping of $\mathrm{VO}_{2}$ nanostructures with aluminum is proposed, which renders possible stabilization of the monoclinic M2 phase in free-standing nanoplatelets and opens an opportunity for realization of a purely electronic Mott transition field-effect transistor without an accompanying structural transition [98].

The aforesaid signifies that the task of creating of the $\mathrm{VO}_{2}$-based MTFET with dimensions down to $10 \mathrm{~nm}$ or less looks quite realistic, inasmuch as the substantially electronic nature of the MIT provides the possibility of the transition triggered by the electric field, and not by temperature. In the next section, we therefore investigate the problem of electronic control the insulator-to-metal transition in vanadium dioxide.

\section{Electronic Control of the MIT at Switching AND INJECTION}

In vanadium dioxide, as well as in a number of other TMOs [10, 28, 51, 52], the switching effect is associated with the development of an insulator-to-metal transition in the electric field [68]. The proposed switching mechanism [68] is based on an electronically induced MIT occurring in conditions of the non-equilibrium carrier density excess under the applied electric field $E$ due to field-stimulated donor ionization, i.e. the Poole-Frenkel effect:

$$
n=N_{0} \exp \left(-\frac{W-\beta \sqrt{E}}{k T}\right),
$$

where $N_{0}$ is a constant independent of the field and only slightly dependent on temperature, $W$ - the conductivity activation energy, and $\beta=\left(e^{3} / \pi \varepsilon \varepsilon_{0}\right)^{1 / 2}$ - the Poole-Frenkel constant. This leads to the elimination of the Mott-Hubbard energy gap at $T_{0}<T_{\mathrm{t}}$ with $T_{0}$ being the ambient temperature. This effect may be treated also as a lowering of $T_{\mathrm{t}}$ due to an excess negative charge (electrons). This model not only allows the qualitative description of the switching mechanism, but it is in quantitative agreement with the experimental results, in particular, with those concerning the critical concentration and the $T_{\mathrm{t}}(E)$ dependence. The model takes into account the dependence of the carrier density on electric field, as well as the dependence of the critical electric field on carrier density, i.e. the scaling of the critical field [68].

These results [68], evidencing of a decrease of $T_{\mathrm{t}}$ in the applied electric field, attach especial importance to the problem of direct influence of electronic effects upon the MIT in vanadium dioxide. The field effect upon the MIT in $\mathrm{VO}_{2}$ has been studied previously, both theoretically and experimentally, in a number of works [99]-[105]. Particularly, a thermodynamic analysis based on the standard phenomenological approach [99], [104], using the equation for the free energy, shows that the shift of the transition temperature in electric field is

$$
\left|\Delta T_{\mathrm{t}}\right| \approx T_{\mathrm{t}} \varepsilon \varepsilon_{0} E^{2} / q,
$$

where $\varepsilon \varepsilon_{0}$ is the material dielectric permittivity, and $q$ - the transition latent heat. The change in $T_{\mathrm{t}}$ is negligible in this case $\left(\sim 1 \mathrm{~K}\right.$ for $\left.E=10^{5} \mathrm{~V} / \mathrm{cm}\right)$. Also, since the entropy of $\mathrm{VO}_{2}$ increases at the transition into metallic phase, the value of $T_{\mathrm{t}}$ increases with increase in $E$, i.e. $\Delta T_{\mathrm{t}}<0$ in Equation (5) [99]. A decrease in $T_{\mathrm{t}}$ in an electric field, and finally its fall down to zero at a certain critical field $E_{\mathrm{c}}$, can be obtained using a microscopic, not thermodynamic, approach based on the detailed MIT mechanism. Unfortunately, as noted in [99], we have no quantitative theory to describe such a transition.

It should be noted that the experiments in MOS structures with $\mathrm{VO}_{2}$ as the semiconductor (which is what the configuration of structures studied most often) under the conditions of a static field effect may turn out to be ineffective, since the creation of a surface potential providing a significant increase in charge carriers density, in a sufficiently thick layer, will probably require an applied gate voltage $V_{\mathrm{G}}$ comparable to the dielectric breakdown voltage. In addition, the non-equilibrium charge density in the enhancement mode in vanadium dioxide declines rapidly with depth because of the relatively high conductivity (low Debye screening length) of the material. That is, such a classical field effect on $T_{\mathrm{t}}$ in $\mathrm{VO}_{2}$ appears to be rather impracticable or unobservable if not impossible at all. A more promising approach, to our understanding, is based not on the field effect, but on the carrier injection from outside.

In our experiments described in [44] we carried out the injection of electrons from $\mathrm{Si}$ into $\mathrm{VO}_{2}$ in the structures Si$\mathrm{SiO}_{2}-\mathrm{VO}_{2}$ (Fig. 9) on p-type silicon with $\rho=0.1 \Omega \cdot \mathrm{cm}$ and $d_{\mathrm{I}}=70 \mathrm{~nm}$ (the $\mathrm{SiO}_{2}$ layer thickness). These experiments have shown that the non-equilibrium electrons injected 
from the $\mathrm{Si}$ substrate can initiate switching - and, consequently, the MIT - in $\mathrm{VO}_{2}$ at a certain critical electron density $n_{\mathrm{c}}$.

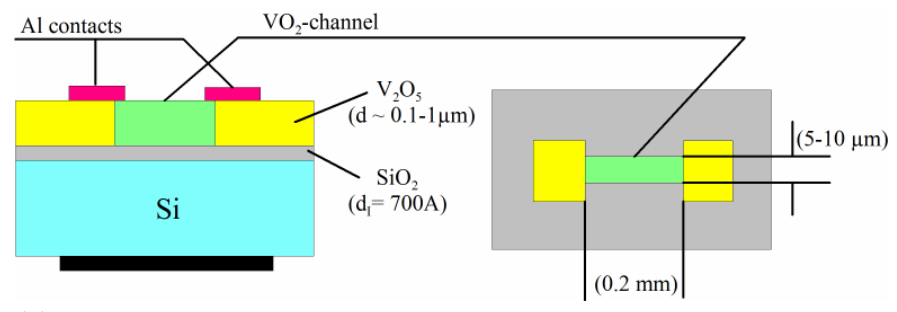

(a)

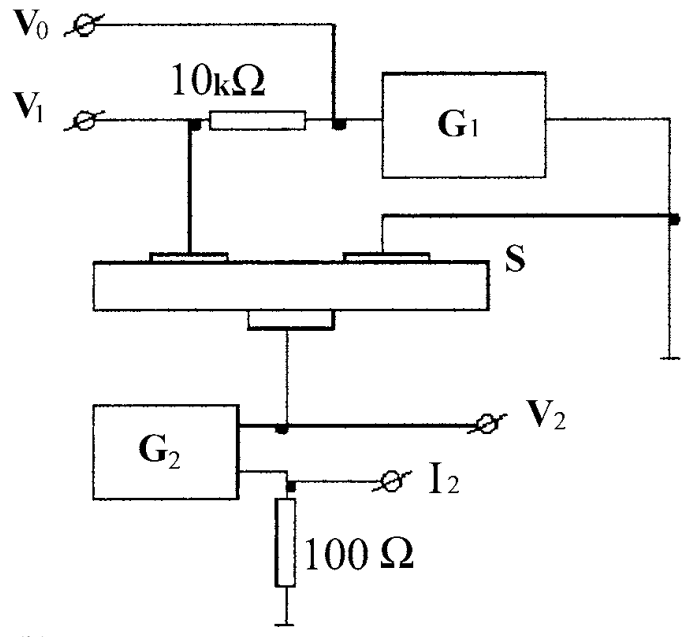

(b)

Fig. 9. (a) Schematic $\mathrm{Si}-\mathrm{SiO}_{2}-\mathrm{VO}_{2}$ structure described in [44] and (b) the injection experiment set-up: $\mathrm{G}_{1}-$ square pulse generator, $\mathrm{G}_{2}-$ ramp generator (maximum amplitude $\sim 100 \mathrm{~V}$ ), $\mathrm{S}$ - structure under study (see panel (a)). The voltages $V_{0}, V_{1}$ and $V_{2}$, and the current $I_{2}$ were measured by a two-beam four-input oscilloscope.

The value of $n_{\mathrm{c}}$ was estimated as follows. The total charge passing through the structure is approximately equal to $I_{a} \cdot \tau_{a}$, where $I_{\mathrm{a}}$ and $\tau_{\mathrm{a}}$ are, respectively, the current and duration of the avalanche injection pulse. For one of the samples, the channel volume $\Omega$ of the planar switching structure (Fig. 9, a) was measured to be $\Omega=1.3 \cdot 10^{-10} \mathrm{~cm}^{3}$, the current was $I_{a}=0.9 \mathrm{~mA}$, and $\tau_{a} \sim 100 \mathrm{~ns}$ (see Fig. 10). Thus, the charge density injected from $\mathrm{Si}$ into $\mathrm{VO}_{2}$ was calculated to be:

$$
n_{\mathrm{inj}}=I_{a} \tau_{a} / e \Omega=4.3 \cdot 10^{18} \mathrm{~cm}^{-3} .
$$

This value by the order of magnitude is equal to the carrier density in the semiconducting phase of $\mathrm{VO}_{2}$ at $T \rightarrow T_{\mathrm{t}}: n_{\mathrm{s}} \sim 10^{18}-10^{19} \mathrm{~cm}^{-3}$ [9], [44]. It is important to note that the value of $n_{\mathrm{c}}\left(\sim n_{\mathrm{inj}}\right)$ is much less than the electron density in the metallic phase of $\mathrm{VO}_{2}\left(n_{\mathrm{m}}=10^{22}-10^{23} \mathrm{~cm}^{-3}\right)$. Therefore, the obtained results may not be accounted for merely by a sharp increase in the switching channel conductivity due to the pumping up of a sufficiently high carrier density to the conductivity band of the material.

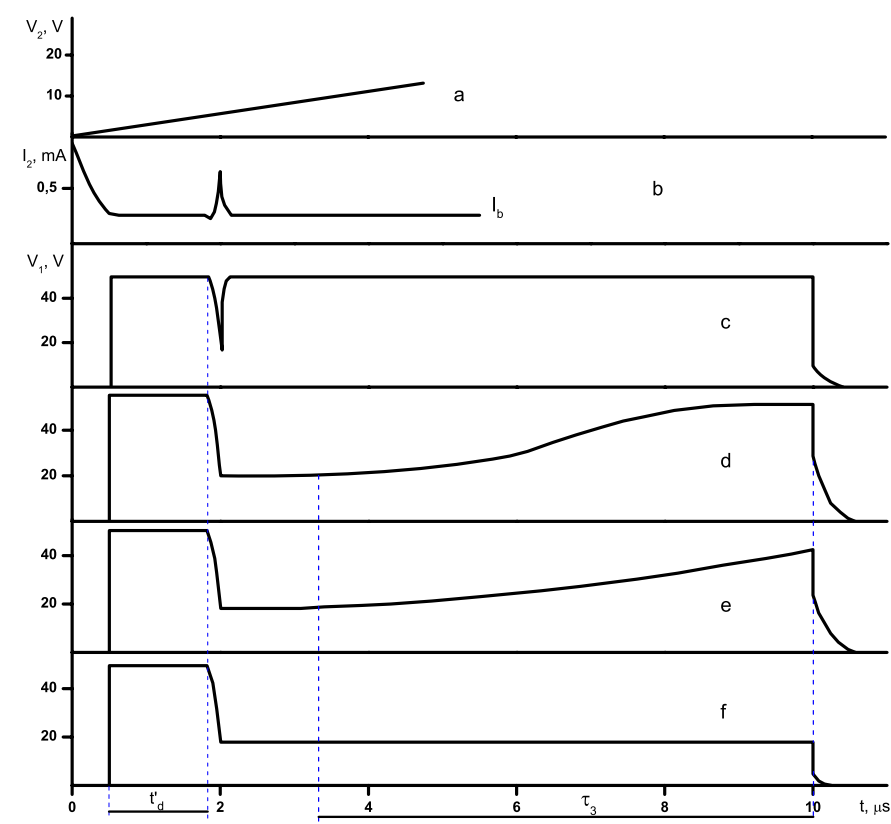

Fig. 10. Injection oscillograms [44]: (a) the substrate voltage $\left(V_{2}\right)$; (b) the current in the substrate circuit, and $I_{\mathrm{b}}$ is the bias current due to the capacity charging by the voltage $V_{2}$; the peak in $I_{2}$ corresponds to the injection pulse and its amplitude and duration are, respectively, $I_{a}$ and $\tau_{a}$. The panels (c) to (f) represent voltage pulses across the $\mathrm{VO}_{2}$ channel at different values of $V_{\text {o }}$ (see Fig. 9, b): $V_{\text {oc }}<V_{\text {od }}<V_{\text {oe }}<V_{\text {of }} ; t_{\mathrm{d}}$ is the switching delay time, and $\tau_{3}-$ the recovery time.

Thus, in $\mathrm{VO}_{2}$ the MIT occurs at a certain $n=n_{\mathrm{c}}$, and it does not matter what is the way of the initiation of this transition - either under heating up to $T=T_{\mathrm{t}}$ (i.e. as the result of the equilibrium thermal generation of carriers), or under photo-generation [63], [64], [86]-[91], injection [44], or high-field generation at switching [51], [52], [68], [92][94]. The transition at $n=n_{\mathrm{c}}\left(T<T_{\mathrm{t}}\right)$ is equivalent to the fact that the value of $T_{\mathrm{t}}$ decreases with increasing electron density [68].

For the electron correlation-induced MIT mechanism the value of $n_{\mathrm{c}}$ can be estimated from the Mott criterion (1) whence for $\mathrm{VO}_{2}$ one obtains:

$$
n_{\mathrm{c}}=\left(0.25 / a_{\mathrm{H}}\right)^{3} \approx 3 \cdot 10^{18} \mathrm{~cm}^{-3}
$$

The Bohr radius is calculated here from Equation (2), and for $\varepsilon=100$ and $m^{*}=3 m_{\mathrm{e}}$ [44] this yields $a_{\mathrm{H}}=1.8 \mathrm{~nm}$ in vanadium dioxide. Note that this quantity of $n_{\mathrm{c}}$ in (7) practically coincides with the above estimate of $n_{\text {inj }}(6)$. Consequently, the electron injection results in exactly the phase transition, and the estimate of the critical density $n_{\mathrm{c}}=n_{\mathrm{s}} \approx 10^{18}-10^{19} \mathrm{~cm}^{-3}$ can be considered as a satisfactory approximation which supports the Mott-Hubbard character of the MIT in $\mathrm{VO}_{2}$.

As was noted in the beginning of this section, the pure field effect in $\mathrm{VO}_{2}$ has been originally studied in planar MIS structures with $\mathrm{SiO}_{2}$ or mica as insulating layers [102], [103]. These experiments demonstrated that the external 
electric field could affect the characteristics of switching (and, hence, of the MIT), yet the effects were rather weak and might not be interpreted in terms of a direct influence of the electric field upon the MIT. Alternative explanations were based on either the inverse piezoelectric effect or an additional Joule heating of the material by leakage currents passing through the gate insulators.

The thermal effects can be excluded if we study the field effect in planar structures $\mathrm{Si}-\mathrm{SiO}_{2}-\mathrm{Si}_{3} \mathrm{~N}_{4}-\mathrm{VO}_{2}$. The carrier injection via silicon oxide into nitride would result in the accumulation of a sufficiently large charge, and then, provided that the nitride layer is thin enough, the trapped charge would create an electric field on the $\mathrm{Si}_{3} \mathrm{~N}_{4}$ outer surface, i.e. on the $\mathrm{Si}_{3} \mathrm{~N}_{4}-\mathrm{VO}_{2}$ interface. This field, in turn, would affect directly upon the electron subsystem of vanadium dioxide shifting its transition temperature (Fig. 11).

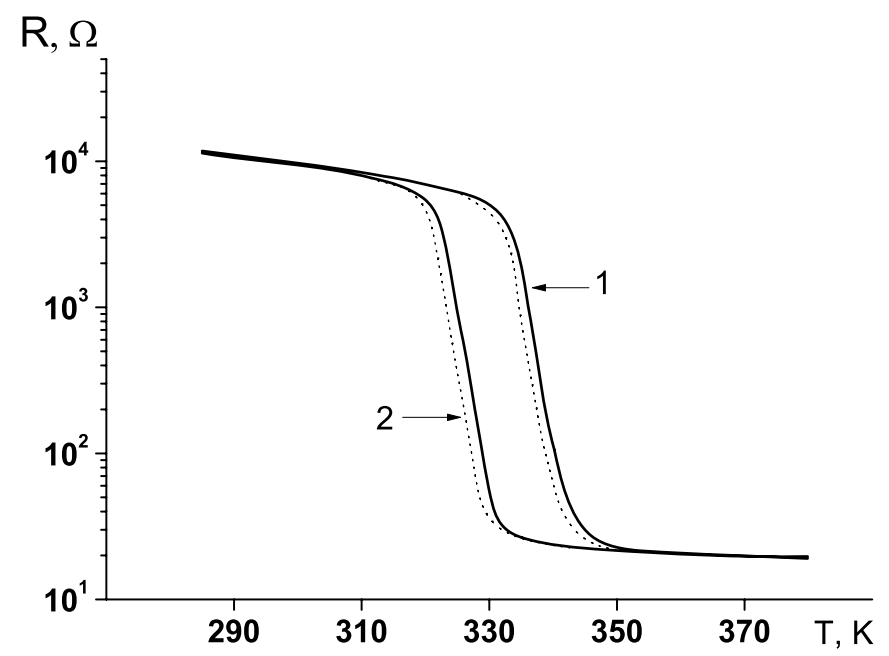

Fig. 11. Temperature dependence of the $\mathrm{VO}_{2}$ film resistance: initial curve (1) and after charge accumulation in the $\mathrm{Si}_{3} \mathrm{~N}_{4}$ sublayer (2) [105].

The experiments [105] were performed with the structures of two types (A and B). The samples of type A were formed on $\mathrm{n}-\mathrm{Si}$ substrates with the oxide and nitride layer thicknesses of 60 and $100 \mathrm{~nm}$, respectively. The type B samples were formed on p-Si with 50-nm-thick $\mathrm{SiO}_{2}$ and 100-nm-thick $\mathrm{Si}_{3} \mathrm{~N}_{4}$. The $\mathrm{VO}_{2}$ layers were deposited onto the nitride surfaces, and the entire structures were completed by thermally evaporated $\mathrm{Al}$ electrodes. The structures were tested by measuring the $C-V$ characteristics and the conductivity jump at the MIT in $\mathrm{VO}_{2}$.

The samples were studied in the regime of tunnel injection of electrons (type A) or holes (type B) and their accumulation in the nitride layers. The injection voltage pulse $\left(V \leq 110 \mathrm{~V}, t=0.1 \mu \mathrm{s}, f=10^{3} \mathrm{~Hz}\right)$ of a corresponding polarity was applied to the bottom (substrate) metal electrode. The charge accumulation was monitored by a shift of the flat-band voltage of the $C-V$ curves $\Delta V_{\mathrm{FB}}$. The value of $\Delta V_{\mathrm{FB}}$ depended on the substrate type, the amplitude and duration of the pulse, and was measured to be in the range $\sim 6-12 \mathrm{~V}$. Next, the conductivity temperature dependences of the $\mathrm{VO}_{2}$ film were measured by the four-probe method. No electric field effect was observed in the structures of type A, while the samples of type B exhibited a shift of $T_{\mathrm{t}}$ toward lower temperatures (Fig. 11).

The results presented [44], [68], [105] confirm thus the influence of excess electrons on the MIT under the field effect conditions in $\mathrm{VO}_{2}$, i.e. the possibility of a transition of vanadium dioxide from a semiconductor state to a metallic state under the action of an electric field and, thereby, the possibility of the $\mathrm{VO}_{2}$-based MTFET implementation.

\section{MotT TRANSITION FET}

\section{A. Vanadium Dioxide}

The idea of a transistor based on the MIT in $\mathrm{VO}_{2}$ had first been advanced [99] in 2002, though with a rather pessimistic outlook because of a negligible modulation of $T_{\mathrm{t}}$ (according to the estimate by the authors of [99] - see Equation (5)). Even earlier, in the work on the electron injection effect upon the MIT [44] we have also discussed this possibility on the basis of the MTFET idea introduced in [42]. Since about 2010 the research in this area has noticeably intensified (see [106]-[118]) and a lot of really encouraging results have been obtained, the review of which is presented below; some preliminary overviews one can also find in the recent surveys [23], [46], [116], [117].

For the first time, the experiments on "pure" field effect in a thin-film transistor structure based on vanadium dioxide have apparently been performed in the work [106]. The essence of this FET on $\mathrm{VO}_{2}$ is presented in Fig. 12, and the experimental results obtained in [106] are reproduced in Fig. 13. One can see that the change in the channel resistance is not very great: about $100 \Omega$ of $2 \mathrm{k} \Omega$, see Fig. 13 (c, d), i.e., $\sim 5 \%$ at $V_{\mathrm{G}}=10 \mathrm{~V}$. This is due precisely to a low thickness of the SCR (space charge region) enriched by electrons as compared with the total film thickness, as discussed above in Section III. Similar results (the MIT-induced channel impedance modulation is only $6 \%$ ) have been obtained in [108], where the gate dielectric is $\mathrm{HfO}_{2}$, not $\mathrm{SiO}_{2}$. That is, in spite of the fact that the resistivity jump at the MIT in $\mathrm{VO}_{2}$ is 4-5 orders of magnitude, the resistance of a thin metal channel with the rest insulating ballast volume connected in parallel varies only within a few percent.

The estimates of the Debye screening length (and hence the corresponding SCR width) reported in [79] give $L_{\mathrm{d}}$ for the semiconductor $\mathrm{VO}_{2}$ phase in the range of 3 to $90 \mathrm{~nm}$. A more detailed calculation [109] shows that $L_{\mathrm{d}}=8 \mathrm{~nm}$, and the thickness of the $\mathrm{VO}_{2}$ layer where the carrier density reaches the critical value $n_{\mathrm{c}}$ is only $0.9 \mathrm{~nm}$ (for the electric field strength magnitude at the $\mathrm{SiO}_{2}-\mathrm{VO}_{2}$ interface of $1.6 \cdot 10^{5} \mathrm{~V} / \mathrm{cm}$ ), although these calculations are to be refined, in particular, taking into account the new data on the vanadium dioxide electronic band structure [110]. 

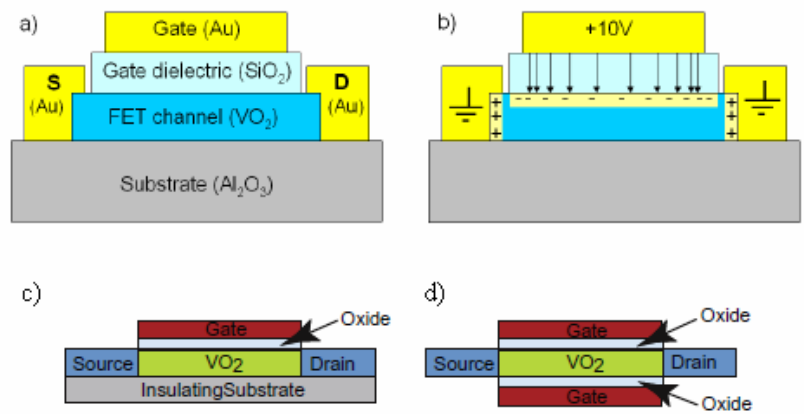

Fig. 12. The method of the $\mathrm{VO}_{2}$-based MTFET realization: a) scheme and b) creation of an electron-enriched metal layer in $\mathrm{VO}_{2}$ in the enhancement mode [118]. Schematic representation of the same from the work [107] (c) and double-gated structure (d).
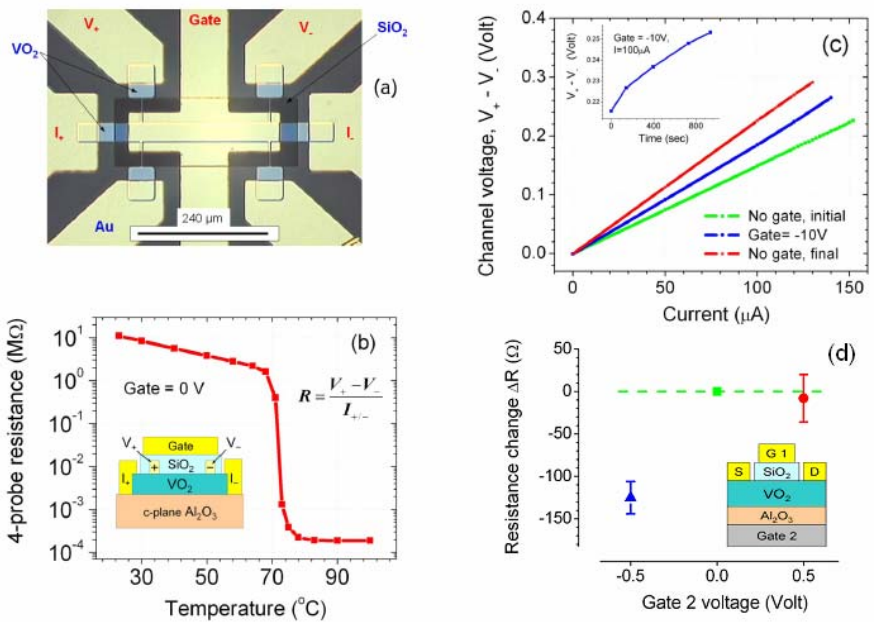

Fig. 13. Experimental results attained in the work [106] (reproduced from [118]): (a) Structure under study (see Fig. 12, a); (b) temperature dependences of the channel resistance; (c) the source-drain $I-V$ characteristics at different gate voltages and (d) the system parameters for the case of a double-gated structure, at different gate-1 voltages, the same as in panel (c).

It is quite evident that the gain in the output characteristic modulation percentage, when altering the gate voltage, can be achieved in this situation by reducing the film thickness $d$ of vanadium dioxide. Mathematical modeling of the $\mathrm{VO}_{2}$-MTFET operation for $d=1.5 \mathrm{~nm}$ (Fig. 14), carried out in [107], shows that in this case quite acceptable parameters (in particular, the limit response time $t_{\mathrm{d}}=0.5 \mathrm{ps}$ at the threshold power $W_{\text {th }} \sim 0.1 \mu \mathrm{W}$ ) can be obtained. However, according to the authors of [107], these limits are difficult to achieve in a real device because of the restrictions associated with the MIT kinetics and heat dissipation; a more realistic estimate gives $t_{\mathrm{d}}=2 \mathrm{ps}$ and $W_{\mathrm{th}}=1-10 \mu \mathrm{W}$. Note that the above discussed purely electronic nature of the MIT in $\mathrm{VO}_{2}$ appears to remove such a concern, because the heat effects would be negligible in this case.

Another problem is connected with the question of whether the MIT will ever exist in such a thin $(1-2 \mathrm{~nm})$ film of $\mathrm{VO}_{2}$, because the value of $d \sim \xi_{1}$ [67], [97] (see Section II.B) is close to the theoretical limit of the influence of dimensional effects on the transition. However, as was

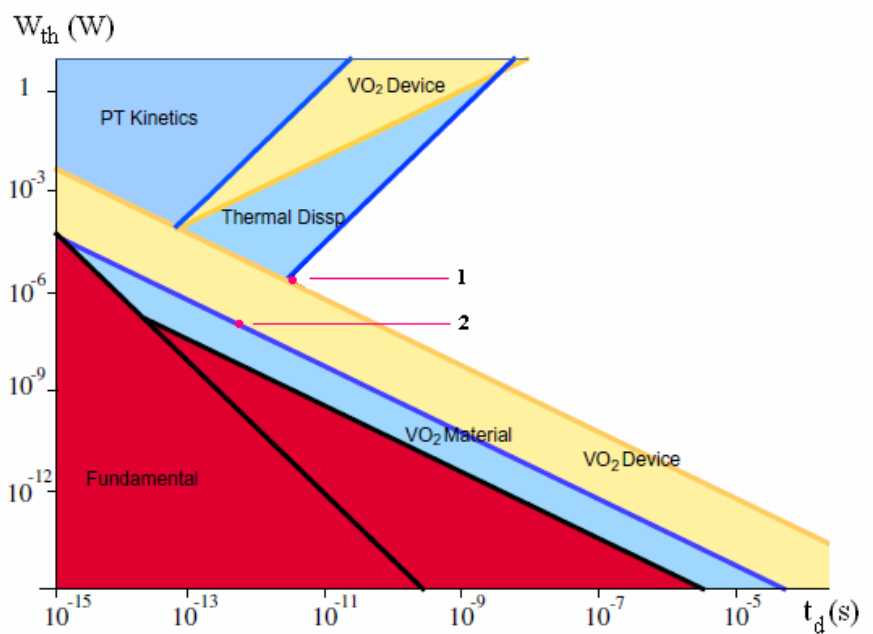

Fig. 14. Power-time diagram for the $\mathrm{VO}_{2}$-MTFET operation [107]. The product of $W$ and $\left(t_{\mathrm{d}}\right)^{2}$ has a quantum restriction (the Planck constant) and this area is denoted as «Fundamental», and the restrictions connected with the phase transition kinetics and thermal dissipation are also shown in the upper area. The analysis has been carried out [107] with the following parameters: $V_{\mathrm{G}}=0.5 \mathrm{~V}$, channel size $\Omega=1.5 \times 50 \times 3 \mathrm{~nm}^{3}$; points 1 and 2 represent, respectively, optimal regimes for $\mathrm{VO}_{2}$ device and $\mathrm{VO}_{2}$ material.

discussed in Section II.C, the study of the properties of nanostructured $\mathrm{VO}_{2}$ shows that the MIT is not suppressed at the transition to nano-scale down to $10 \mathrm{~nm}$ and even perhaps below.

It is known that oxide semiconductor systems can be electrostatically doped with organic ionic liquids as gate insulators in order to modulate their carrier density and thereby induce phase transitions [111]. This results from the charge accumulation in the double layer at the electrolyte-oxide interface, and the electric field of this charge creates an electron-enriched layer in $\mathrm{VO}_{2}$ which leads to the MIT, i.e. this approach is similar to that described above in the end of Section III (Fig. 11) where the charge accumulation occurs in the silicon nitride film. The channel conductance change has been reported to be up to $50 \%$ at a voltage $V_{\mathrm{G}}=+2 \mathrm{~V}$ [111], and the work [112] reports on complete metallization throughout the $\mathrm{VO}_{2}$-FET channel, not only a near-gate layer. However, it has been shown [113] that this effect is related to the fieldinduced extraction of oxygen and the formation of oxygen vacancies which, in turn, being the donor type stoichiometry defects, contribute to an increase in free carrier concentration resulting finally in the transition into a metallic phase according to the Mott mechanism (similar to the way as it occurs due to the hydrogen injection [75]-[77], [119]).

In the work [114], the voltage-induced MIT in $\mathrm{VO}_{2}$ nanoparticles on a $\mathrm{SiO}_{2} / \mathrm{Si}(100)$ substrate has been investigated. The results reveal that $T_{\mathrm{t}}$ decreases by $\sim 0.48$ and $\sim 0.87^{\circ} \mathrm{C} / \mathrm{V}$ in the heating and cooling processes, respectively. Also, the authors of [115] have explored the MIT in $\mathrm{VO}_{2}$-based tunnel junction. It is shown that, in such tunnel field effect transistors, the MIT can be utilized to 
modulate the tunnelling current by opening an energy gap in the OFF-state, and by collapsing the gap in the ON-state. It is also to be noted that some ideas, related to the use of materials with MIT, including $\mathrm{VO}_{2}$, for a FET device construction, have been patented (see for example [42], [120]).

The main conclusion that can be drawn from the analysis presented in this section is as follows. The main difficulty in implementing the $\mathrm{VO}_{2}$-based MTFET is a small thickness of the metal phase layer, and as a consequence, the low magnitudes of the impedance jump at the MIT (in spite of the fact that the $\mathrm{VO}_{2}$ conductivity jump can reach five orders of magnitude) under the influence of an applied electric field. In our opinion, there exist the following possible ways to overcome this problem:

1) Use thinner films of vanadium dioxide (e.g., $5 \mathrm{~nm}$, and the SCR width of $1 \mathrm{~nm}$ will be equal in this case to almost one fifth of the total thickness).

2) Double-gate structures (Figures 12, d and 13, d) [106], [107].

3) Doping (e.g., with tungsten) in order to reduce $T_{\mathrm{t}}$, and thereby to diminish the quantity of necessary additive to electron density to achieve the value of $n_{\mathrm{c}}$; however, an optimal doping level should be carefully adjusted for the avoidance of the possible complete suppression of the MIT under doping, on the one part, and on the other part, for the increase in the semiconducting phase resistivity, but so as to retain the resistivity jump at the MIT as high as possible.

In addition, the field effect on the MIT in $\mathrm{VO}_{2}$ could be enhanced by varying the temperature so as to either achieve a higher contrast between the resistivities of the semiconducting and insulating phases (at cooling) or approach to the transition onset (at heating). The same result might be obtained at room temperature by dint of, for example, proper doping, like in item 3 above, but it is to be stressed that the way of temperature variation is suitable only for investigation, i.e. in order to elucidate the problem, and not for device operation, since in this case, such an advantage of $\mathrm{VO}_{2}$ as room temperature compatibility is in fact lost.

\section{B. Other Materials}

Aside from vanadium dioxide, similar experiments in the area of MTFET are also carried out with some other materials [12]-[15], [46], [95], [96], [116], [121]-[132], such as, e.g., the Mott insulator $\mathrm{GaTa}_{4} \mathrm{Se}_{8}$ [121], [127]. The principle of FET operation in this case is the same as for $\mathrm{VO}_{2}$, with the only difference being that the mechanism of a high-field charge carrier augmentation up to the value of $n=n_{\mathrm{c}}$ may be other than the Poole-Frenkel donor ionization (as it takes place in $\mathrm{VO}_{2}$ [68], [72], [82], [107], [133] [135]), and it may be, for instance, the Zener or avalanche breakdown mechanism. Particularly, for $\mathrm{GaTa}_{4} \mathrm{Se}_{8-\mathrm{x}} \mathrm{Te}_{\mathrm{x}}$ it has been shown [130] that the breakdown threshold voltage depends on the Mott-Hubbard gap width as $E_{\mathrm{th}} \sim\left(E_{\mathrm{g}}\right)^{5 / 2}$ which is characteristic of avalanche breakdown in semiconductors.

Various materials for oxide electronics, basically strongly correlated TMOs, are discussed in the reviews [23], [46], [116], [117], where not only FETs, but also other electronic devices are considered, in particular, neurochips and neural networks on the basis of memristors [117], [136], including those made of TMO-based switching elements.

One more material proposed for MTFET is magnetite $\left(\mathrm{Fe}_{3} \mathrm{O}_{4}\right)$ [46], [95], [116], [122], [132] exhibiting the Verwey transition at $T_{\mathrm{t}}=120 \mathrm{~K}$ [47]. It has been shown [122] that the transition in magnetite in the applied electric field is due to the field effect and not caused by local Joule heating. Also, the Verwey transition has been found to be a two-step process with characteristic time scales of $300 \mathrm{fs}$ and $1.5 \pm 0.2$ ps which establishes the speed limit for oxide electronics devices based on magnetite [132].

It should be noted that the oxide materials are not the only candidates for the MTFET realization. In addition to the aforementioned $\mathrm{GaTa}_{4} \mathrm{~S}_{8}$ [121], [127], [130], molybdenum disulfide can serve as an example [123], [131]. In particular, 2D materials are a new class of materials with interesting physical properties and applications ranging from nanoelectronics to sensing and photonics [131]. In addition to graphene, the most studied 2D material, monolayers of semiconducting dichalcogenides $\mathrm{MoS}_{2}$ or $\mathrm{WSe}_{2}$ are gaining in importance as promising channel materials for FETs. High levels of doping achieved in dual-gate devices have allowed the observation of a metal-insulator transition in monolayer $\mathrm{MoS}_{2}$ due to strong electron-electron interactions [131]. Also, quasi-1D organic compounds are examined too; for example, a field effect transistor structure using chargeordered organic materials $\alpha$-(BEDT-TTF) $)_{2} \mathrm{I}_{3}$ and $\alpha^{\prime}$-(BEDT-TTF) ${ }_{2} \mathrm{IBr}_{2}$ has been recently reported [124].

Amongst other materials, multicomponent perovskite-like oxides are worthy to be mentioned. For example, a Mott-transition FET that exhibits a switching ratio of more than two orders of magnitude at room temperature has been demonstrated by using the electric double layer of an ionic liquid for gating on a strongly correlated electron system $\mathrm{SmCoO}_{3}$ [129], an electroninduced MIT in a two-terminal device based on $\mathrm{NdNiO}_{3}$ has been reported in [125], and MTFET prototypes based on $\mathrm{CaMnO}_{3}$ and $\mathrm{NdNiO}_{3}$ are described in [23]. In the brief note [128] the author compares FETs based on strongly correlated TMOs and those on $\mathrm{Si}$. The devices on the basis of $\mathrm{SmNiO}_{3}, \mathrm{VO}_{2}$, and perovskite-based sketch-FETs (see Section I and references [11]-[16]) are examined. As for the vanadium dioxide, it is stated, with the reference to the work [137] that "in $\mathrm{VO}_{2}$ the transition is in large part caused by micrometre-scale heating by the applied electric field". It should be stressed however that thermal effects do take place at switching (not at the field effect) [68], and it has always been known that the voltage-induced MIT in a 
macroscopic $\mathrm{VO}_{2}$ crystal occurs due to the Joule heating effect [138]. Note that a "micrometer-scale" size is surely macroscopic with respect to nano-scale dimensions. Therefore, the results of [137] do not contradict the idea of the field-controlled MIT in $\mathrm{VO}_{2}$.

In conclusion, we point out the main advantages of $\mathrm{VO}_{2}$ compared to the other materials considered as candidates for the implementation of MTFET devices:

1) $T_{\mathrm{t}}$ is close to room temperature, unlike, e.g., $\mathrm{Fe}_{3} \mathrm{O}_{4}$ whose $T_{\mathrm{t}}$ is much lower than $300 \mathrm{~K}$.

2) $\mathrm{VO}_{2}$ is a simple binary oxide which is more technologically convenient and easy to handle as compared to sulfides or complex perovskite oxides. In addition, this compound is comprehensively studied and can easily be obtained by a number of technological routes as thin films and nanostructures.

3) The transition temperature might be finely adjusted by means of proper doping.

\section{CONCLUSIONS AND OUTLOOK}

In the present review, we have discussed some current research, urgent problems and near-term prospect of oxide electronics, one of the new and rapidly developing fields of "beyond-silicon" electronics. Surely, rumors of the forthcoming demise of conventional silicon electronics seem to be heavily exaggerated. However, as we have attempted to show in Section I, alternative approaches, such as spintronics, superconducting electronics, singleelectronics, molecular electronics, soletronics and so forth, are being intensively developed all over the world, and the reasons for this have also been discussed in the introduction. Such terms and acronyms as, for instance, SET (single-transistor electron), RSFQ (rapid single flux quantum) superconducting devices, spin FET, etc. become now as familiar and customary as the MOSFET. Further it has been shown there that one of such novel directions, namely, oxide electronics, is based on the unique properties and physical phenomena, particularly, the metal-insulator transition, in strongly correlated transition metal oxides. Our discussion of oxide electronics has been rather selective and we have confined ourselves to only one, though very important, kind of device, viz., $\mathrm{VO}_{2}$-based Mott-transition FET. We have almost passed over in silence both other areas of oxide electronics (except for a brief mention of those in Section I provided with some references) and other materials, i.e. not only $\mathrm{VO}_{2}$, for the oxide FETs design, yet the latter have been discussed in Section IV.B, and the search for new efficient materials and systems for the MTFET idea realization has been shown to be of importance.

It should be emphasized that the interest in vanadium dioxide, as well as in the problem of metal-insulator transition on the whole, results from not only a possible practical yield, but this interest is of course connected first of all with basic knowledge, since the MIT is one of the most vital and spectacular problems in modern condensed matter physics [9], [10], [43], [46]-[48]. Recently this problem has received considerable attention once again, primarily due to the discoveries of HTSC and CMR phenomena in perovskite-like metal-oxide cuprates and manganites [9], [49]. The MIT has been widely studied over the last years, with special emphasis on the particular case known as the Mott transition [9]. At zero temperature, this process is controlled by the electron density. A simple picture, which is able to grasp the main physical points and to estimate the critical density $n_{\mathrm{c}}$ for the Mott transition to occur, is based on the Mott criterion, which has been briefly discussed in Section II.B. This picture is very qualitative and ignores fundamental aspects of the MIT, such as magnetic effects, electron-phonon coupling (polarons), disorder (Anderson localization) and some other interactions. Nevertheless, various approaches based on the Mott-Hubbard theory, taking the above-mentioned effects into account, successfully describe the MIT in many particular cases, from doped semiconductors to TMOs [9].

The generally known example of the MIT in doped semiconductors (with an increase in the impurity concentration) [9] is far from being the only one. There are a lot of materials (mainly compounds of transition and rareearth metals) exhibiting a temperature-induced MIT [9], [10], [47], [48], likewise any other usual phase transitions superconducting, ferroelectric, or magnetic. No consistent and unified theory of MIT exists for transition-metal compounds, unlike for doped semiconductors, where everything is more or less clear, at least, conceptually. Instead, there are a variety of models: the electroncorrelation Mott-Hubbard transition; the Peierls transition in quasi-one-dimensional systems with charge- or spindensity waves; the Verwey transition (based on the idea of Wigner crystallization of the electron gas) involving charge ordering; the Anderson transition due to the disorderinduced localization, etc. In the absence of accurate theories, MITs in real systems have been explained (often merely qualitatively) in terms of the available models [48]. As we have discussed in this review, strongly correlated transition metal oxides exhibiting such phase transitions are currently considered as basic functional materials of oxide electronics. That is why the theoretical models describing MITs are of such importance, and vanadium dioxide represents a model system for understanding correlated oxides in general and diverse phenomena therein, including such effects as MIT, HTSC, and CMR. For example, the authors of [139] write that their studies of a solid-state triple point between the insulating M1 and M2 and metallic $\mathrm{R}$ phases at the MIT in vanadium dioxide "have profound implications for the mechanism of the metal-insulator transition in $\mathrm{VO}_{2}$, but they also demonstrate the importance of this approach for mastering phase transitions in many other strongly correlated materials, such as manganites and iron-based superconductors".

Further, it is to be noted that, generally, the ability of 
some materials to switch their conductance between two stable states provides the basis for many electronic devices, not only MTFET. Therefore, the phenomena of metalinsulator transition and switching have various potential applications in electronics and related fields of engineering [10], [46], [47]. Particularly, the TMO-based MOM structures demonstrating memory switching have been proposed as potential candidates for both nonvolatile and dynamic random-access memories [21]-[30]. Also, memory effects due to the electric field induced Mott transition (in cerium oxide particularly) have been studied in [140]. In addition, vanadium dioxide can be used as a material for thermochromic coatings, optical filters and controllers, sensors, bolometers, sensitive elements for RF and $\mathrm{THz}$ radiation control, micro-electromechanical systems, plasmonic modulators, access diodes in oxide ReRAM and many other optical and electronic devices [47], [83], [84], [134], [141]-[166]. Many authors note that the high operation lifetime of $\mathrm{VO}_{2}$-based devices (e.g. more than $10^{8}$ cycles without failure for current-controlled switching [167]), as well as the fast switching coupled with room temperature compatibility could lead to further interest in the use of correlated oxide phase transition devices, including those based on vanadium dioxide, for future electronics [168].

As far as to the oxide electronics area applications concerns directly, in conclusion, we would like to outline a prospect of an all-vanadium-oxide IC containing $\mathrm{VO}_{2}$-MTFETs and vanadium-oxide-based memristors (supplied with $\mathrm{VO}_{2}$ switches as access elements [157]) in one chip. It should be stressed that ReRAM devices may be performed on either MIT materials or other vanadium oxides which do not exhibit a MIT. An example of a $\mathrm{V}_{2} \mathrm{O}_{5}$-based memory device is given in our works [28], [169], [170] - see Fig. 15. Various versions of memory devices utilizing the effect of MIT in vanadium oxides has also been discussed in the literature [30], [31], [156], [162], [163], [171], [172]. It is pertinent to quote here also the work [173] where the experiments on memory switching in thin-film $\mathrm{V}_{2} \mathrm{O}_{5}$-based structures have first been described. To interpret the results of these experiments, the authors of [173] put forward a hypothesis of some "glass-to-metal" transition in amorphous vanadium pentoxide. Afterwards, in the literature, this assumption somehow transformed into an idea of a MIT in $\mathrm{V}_{2} \mathrm{O}_{5}$ which was further widely circulated, (with no efforts to delve into the problem), much referred to, and, finally, it has recently been reported on a "MIT in $\mathrm{V}_{2} \mathrm{O}_{5}$ ", as it is asserted in the titles of the articles [174], [175]. Of course, there is no a MIT in vanadium pentoxide, and we have recently published comments [43], [176] concerning this issue.

Finally, as an addition to the above presented idea of allvanadium-oxide electronics, we note also that mechanical memory, i.e. that similar factually to an $\mathrm{HD}$ or $\mathrm{CD}$, based on the MIT in $\mathrm{VO}_{2}$ has been designed [144], [162], and a flexible display is feasible on the basis of the electrolyte-less electrochromic effect in $\mathrm{V}_{2} \mathrm{O}_{5}$-gel films [175]. Note however that the main requirement to the MIT-based oxide electronics is still its ability to be scaled down to nano-dimensions. In this regard we have to mention two more recent works [176], [177] in addition to those discussed in the end of Section II and in [97].

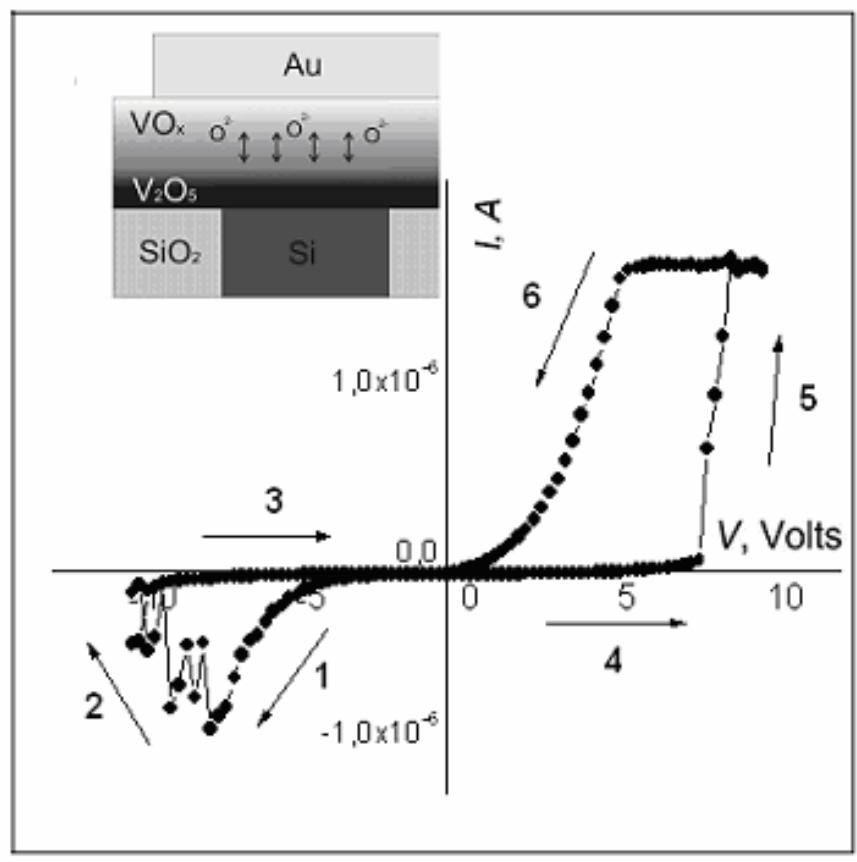

Fig. 15. I- $V$ characteristic of $\mathrm{Au} / \mathrm{V}_{2} \mathrm{O}_{5} / \mathrm{SiO}_{2} / \mathrm{Si}$ junction at room temperature with arrows indicating the direction of the voltage sweep [28], [169]. The structure under study is also depicted schematically.

In conclusion, as to the prospective directions of development of research in the field of MTFET, two recently emerging interesting themes, apart from those discussed in Section IV above, are worthy, in our opinion, of especial mention, namely: a) ionic liquid gating [111], [112], [129], [180] and b) application of MIT-based switches and FETs for design of neurochips [117, 136]. Interestingly, the two, on the face of it, such dissimilar directions seem to find a point of intersection [181].

\section{ACKNOWLEDGMENT}

The authors thank P. Boriskov, A. Cheremisin and V. Putrolaynen for useful discussions, and M. Belyaev for his help with the manuscript preparation.

\section{REFERENCES}

[1] J. S. Kilby. Turning Potential into Reality: The Invention of the Integrated Circuit. Nobel Lecture, 2000 [Online]. Available: http://www.nobelprize.org/nobel_prizes/physics/laureates/2000/kilby -lecture.html.

[2] Yu. V. Gulyaev, V. B. Sandomirskiı̌, A. A. Sukhanov, and Yu. Ya. Tkach, "Physical limitations on miniaturization in microelectronics," Sov. Phys. Usp., vol. 27, pp.868-880, 1984.

[3] H. Takagi and H. Y. Hwang, "An Emergent Change of Phase for Electronics," Science, vol. 327, pp. 1601-1602, 2010.

[4] E. J. Lerner, "The end of the road for Moore's law," Think Research, vol. 4. IBM Thomas Watson Research Center, USA, pp.7-11, 1999; 
see also: Beyond3D Forum [Online]. Available: http://beyond3d.com/showthread.php?t=4750\&page $=8$.

[5] M. Bohr and K. Mistry. INTEL's Revolutionary 22-nm Transistor Technology, 2011 [Online]. Available:

http://download.intel.com/newsroom/kits/22nm/pdfs $/ 22 \mathrm{~nm}$ Details Presentation.pdf.

[6] S. Migita, Y. Morita, M. Masahara, and H. Ota, "Fabrication and demonstration of 3-nm-channel-length junctionless field-effect transistors on silicon-on-insulator substrates using anisotropic wet etching and lateral diffusion of dopants," Jpn. J. Appl. Phys., vol. 52, pp. 04CA01-5, 2013.

[7] S. Zaima, "Technology evolution for silicon nanoelectronics: postscaling technology," Jpn. J. Appl. Phys., vol. 52, p.030001, 2013.

[8] J. F. Rossier, "Single-atom devices: Quantum engineering," Nature Materials, vol. 12, pp. 480-481, 2013; C. Schirm, M. Matt, F. Pauly, J. C. Cuevas, P. Nielaba, and E. Scheer, "A current-driven singleatom memory," Nature Nanotechnology, vol. 8, pp. 645-648, 2013.

[9] N. F. Mott, Metal-Insulator Transition, 2nd ed. London: Taylor and Francis, 1990.

[10] A. L. Pergament, G. B. Stefanovich, A. A. Velichko, and S. D. Khanin, "Electronic Switching and Metal-Insulator Transitions in Compounds of Transition Metals," in Condensed Matter at the Leading Edge. Nova Science Publishers, 2006, pp.1-67.

[11] A. P. Ramirez, "Oxide Electronics Emerge," Science, vol. 315, pp. 1377-1378, 2007

[12] C. Cen, S. Thiel, J. Mannhart, and J. Levy, "Oxide Nanoelectronics on Demand," Science, vol. 323, pp. 1026-1030, 2009.

[13] P. Irvin, M. Huang, F. J. Wong, T. D. Sanders, Y. Suzuki, and J. Levy, "Gigahertz-frequency operation of a $\mathrm{LaAlO} 3 / \mathrm{SrTiO} 3$-based nanotransistor," Appl. Phys. Lett., vol. 102, p. 103113, 2013.

[14] P. Zubko, S. Gariglio, M. Gabay, P. Ghosez, and J.-M. Triscone, "Interface Physics in Complex Oxide Heterostructures," Annu. Rev. Condens.Matter Phys., vol. 2, pp. 141-165, 2011

[15] H. Y. Hwang, Y. Iwasa, M. Kawasaki, B. Keimer, N. Nagaosa, and Y. Tokura "Emergent phenomena at oxide interfaces," Nature Materials, vol. 11, pp. 103-113, 2012.

[16] G. Herranz, M. Basletić, M. Bibes, C. Carretero, E. Tafra, E. Jacquet, K. Bouzehouane, C. Deranlot, A. Hamzić, J.-M. Broto, A. Barthelemy, and A. Fert," High Mobility in LaAlO3/SrTiO3 Heterostructures: Origin, Dimensionality, and Perspectives," Phys. Rev. Lett., vol. 98, no. 21, pp. 216803-216807, 2007.

[17] S. Pang, Y. Hernandez, X. Feng, and K. Mullen, "Graphene as Transparent Electrode Material for Organic Electronics," Adv. Mater., vol. 23, pp. 2779-2795, 2011.

[18] J. Lewis, "Materials challenge for flexible organic devices," Materials Today, vol. 9, pp.38-45, Apr. 2006.

[19] S. Kumar, B. A. Cola, R. Jackson, and S. Graham, "A Review of Carbon Nanotube Ensembles as Flexible Electronics and Advanced Packaging Materials," Journal of Electronic Packaging, vol. 133, no. 2, p. 020906, 2011

[20] D.H. Blank, D.S. Ginley, M.E. Hawley, S.K. Streiffer, and D.C Paine (ed.), Transport and Microstructural Phenomena in Oxide Electronics, Mat. Res. Soc. Proc., vol. 666, 2001.

[21] Y. Fujisaki, "Review of Emerging New Solid-State Non-Volatile Memories," Jpn. J. Appl. Phys, vol. 52, p. 040001, 2013.

[22] R. Waser, "Resistive non-volatile memory devices", Microelectronic Engineering, vol. 86, pp. 1925-1928, 2009.

[23] H. Akinaga, "Recent Advances and Future Prospects in FunctionalOxide Nanoelectronics: The Emerging Materials and Novel Functionalities that are Accelerating Semiconductor Device Research and Development," Jpn. J. Appl. Phys, vol. 52, p. 100001, 2013.

[24] D. B. Strukov and R. S. Williams, "An ionic bottle for high-speed, long-retention memristive devices," Appl. Phys. A, vol. 102, pp. 1033-1036, 2011.

[25] L. Liu, B. Chen, B. Gao, F. Zhang, Y. Chen, X. Liu, Y. Wang, R. Han, and J. Kang, "Engineering oxide resistive switching materials for memristive device application," Appl. Phys. A, vol. 102, pp. 991996, 2011.

[26] D. Ielmini, R. Bruchhaus, and R. Waser, "Thermochemical resistive switching: materials, mechanisms, and scaling projections," Phase Transitions, vol. 84, no. 7, pp. 570-602, Jul. 2011.

[27] S. Balatti, S. Larentis, D. C. Gilmer, and D. Ielmini, "Multiple Memory States in Resistive Switching Devices Through Controlled
Size and Orientation of the Conductive Filament," Adv. Materials, vol. 25 , no. 10 , pp. $1474-1478,2013$.

[28] A. Pergament, G. Stefanovich, A. Velichko, V. Putrolainen, T. Kundozerova, and T. Stefanovich, "Novel Hypostasis of Old Materials in Oxide Electronics: Metal Oxides for Resistive Random Access Memory Applications," Journal of Characterization and Development of Novel Materials, vol. 4, no. 2, pp. 83-110, 2011.

[29] T. V. Kundozerova, A. M. Grishin, G. B. Stefanovich, and A. A. Velichko, "Anodic Nb2O5 Nonvolatile RRAM," IEEE Trans. Electron Devices, vol. 59, no. 4, pp. 1144-1148, Apr. 2012.

[30] T. Driscoll, H.-T. Kim, B.-G. Chae, M. Di Ventra, and D. N. Basov, "Phase-transition driven memristive system," Appl. Phys. Lett., vol. 95, p. 043503, 2009

[31] R. Xie, C. T. Bui , B. Varghese, Q. Zhang, C. H. Sow, B. Li , and J. T. L. Thong, "An Electrically Tuned Solid-State Thermal Memory Based on Metal-Insulator Transition of Single-Crystalline $\mathrm{VO}_{2}$ Nanobeams," Adv. Funct. Mater., vol. 21, pp. 1602-1607, 2011.

[32] S.J. Pearton, W. T. Lim, E. Douglas, H. Cho, and F. Ren, "Flexible Electronics Based on InGaZnO Transparent Thin Film Transistors," Key Engineering Materials, vol. 521, pp. 141-151, 2012.

[33] J. F. Wager, B. Yeh, R. L. Hoffman, and D. A. Keszler, "An amorphous oxide semiconductor thin-film transistor route to oxide electronics," Curr. Opin. Solid State Mater. Sci., in press, 2013. http://dx.doi.org/10.1016/j.cossms.2013.07.002

[34] J. S. Park, W.-J. Maeng, H.-S. Kim, and J.-S. Park, "Review of recent developments in amorphous oxide semiconductor thin-film transistor devices," Thin Solid Films, vol. 520, pp. 1679-1693, 2012.

[35] D. Keszler, "Oxide electronics: Transistors pick up steam," Nature Materials, vol. 10, pp. 9-10, 2011

[36] E. Fortunato and R. Martins, "Where science fiction meets reality? With oxide semiconductors!” Phys. Stat. Solid., vol. 5, pp. 336 -339, 2011.

[37] R. F. P. Martins, A. Ahnood, N. Correia, L. M. N. P. Pereira, R. Barros, P. M. C. B. Barquinha, R. Costa, I. M. M. Ferreira, A. Nathan, and E. E. M. C. Fortunato, "Recyclable, Flexible, LowPower Oxide Electronics." Adv. Funct. Mater., vol. 23, pp. 21532161, 2013.

[38] M. Suzuki and T. Ami, "A proposal of epitaxial oxide thin film structures for future oxide electronics," Materials Sci. Engineering, vol. B41, pp. 163-174, 1996.

[39] C. G. Granqvist, "Transparent conductors as solar energy materials A panoramic review," Solar Energy Materials \& Solar Cells, vol. 91, pp. 1529-1598, 2007.

[40] J. A. Rogers, T. Someya, and Y. Huang, "Materials and Mechanics for Stretchable Electronics," Science, vol. 327, pp. 1603-1607, 2010.

[41] C. Zhou, D. M. Newns, J. A. Misewich, and P. C. Pattnaik, "A field effect transistor based on the Mott transition in a molecular layer," Appl. Phys. Lett., vol. 70, no. 5, pp. 598-600, 1997.

[42] D.M. Newns, J.A. Misewich, and C. Zhou, "Nanoscale Motttransition Molecular Field Effect Transistor," U.S. Patent YO996-06, 1996.

[43] A. Pergament, G. Stefanovich, N. Kuldin, and A. Velichko, "On the Problem of Metal-Insulator Transitions in Vanadium Oxides," ISRN Condensed Matter Physics, vol. 2013, 2013. Available: http://dx.doi.org/10.1155/2013/960627

[44] G. Stefanovich, A. Pergament, and D. Stefanovich, "Electrical switching and Mott transition in $\mathrm{VO}_{2}$," Journal of Physics: Condensed Matter, vol. 12, no. 41, pp. 8837-8845, 2000.

[45] A. L. Pergament and G. B. Stefanovich, "Phase composition of anodic oxide films on transition metals: a thermodynamic approach," Thin Solid Films, vol. 322, no.1-2, pp. 33-36, 1998.

[46] Z. Yang, C. Ko, and S. Ramanathan, "Oxide Electronics Utilizing Ultrafast Metal-Insulator Transitions," Annu. Rev. Mater. Res., vol 41, pp. 337-367, 2011

[47] A. A. Bugaev, B. P. Zakharchenya, and F. A. Chudnovskii, Metalsemiconductor phase transition and its application, Leningrad: Nauka, 1979, 183 pages.

[48] R. O. Zaitsev, E. V. Kuzmin, and S. G. Ovchinnikov, "Fundamental ideas on metal-dielectric transitions in 3d-metal compounds," Sov. Phys. Usp., vol. 29, pp. 322-342, 1986.

[49] P. A. Cox, Transition Metal Oxides. An Introduction to their Electronic Structure and Properties. Oxford: Clarendon Press, 1992. 
[50] S. V. Kalinin and N. A. Spaldin, "Functional Ion Defects in Transition Metal Oxides," Science, vol. 341, no. 6148, pp. 858-859, Aug. 2013.

[51] A. L.Pergament, G. B.Stefanovich, and F. A.Chudnovskii, "Metalsemiconductor phase transition and switching effect in oxides of transition metals," Phys. Solid State, vol. 36, no. 10, pp. 1590-1597, 1994

[52] F. A. Chudnovskii, L. L. Odynets, A. L. Pergament, and G. B. Stefanovich, "Electroforming and switching in oxides of transition metals: The role of metal-insulator transition in the switching mechanism," J. Solid State Chem., vol.122, pp. 95-99, 1996.

[53] N. A. Tulina, "Colossal electroresistance and electron instability in strongly correlated electron systems," Phys. Usp., vol. 50, pp. 1171-1178, 2007.

[54] F. J. Morin, "Oxides which show a metal-to-insulator transition at the Neel temperature," Phys. Rev. Lett., vol. 3, pp. 34-36, 1959.

[55] N. F. Mott, E. A. Davis, Electronic Processes in Non-Crystalline Materials, 2nd ed. Oxford: University Press, 1978

[56] A. D. Wadsley, "Nonstoichiometric Metal Oxides. Order and Disorder." Nonstoichiometric Compounds, vol. 39, ch. 2, pp . 23-36, 1963.

[57] A. Pergament, G. Stefanovich, O. Berezina, and D. Kirienko, "Electrical conductivity of tungsten doped vanadium dioxide obtained by the sol-gel technique," Thin Solid Films, vol. 531, pp. 572-576, 2013.

[58] K. Shibuya, M. Kawasaki, and Y. Tokura, "Metal-insulator transition in epitaxial $\mathrm{V}_{1-\mathrm{x}} \mathrm{W}_{\mathrm{x}} \mathrm{O}_{2}(0<=\mathrm{x}<=0.33)$ thin films," Appl. Phys. Lett., vol. 96, pp. 022102, 2010.

[59] C. N. Berglund and A. Jayaraman, "Electric properties of $\mathrm{VO}_{2}$ near the semiconductor-metal transition," Phys. Rev, vol. 185, no. 3, pp. 1022-1033, 1969; "Hydrostatic pressure dependence of the electronic properties of $\mathrm{VO}_{2}$ near the semiconductor-metal transition temperature," Ibidem, pp. 1034-1039, 1969

[60] R. M. Wentzcovitch, W. W. Schulz, and P. B. Allen, "VO2: Peierls or Mott-Hubbard? A view from band theory," Phys. Rev. Lett., vol. 72, pp. 3389-3392, 1994.

[61] A. L. Semenov, "DC electric-field-induced heterophase structure on the surface of a peierls metal," Physics of the Solid State, vol. 42, issue 6, pp. 1160-1163, Jun. 2000; A. L. Semenov, "About superlattice of phases in vanadium dioxide film," ibidem, vol. 55, issue 12, Dec. 2013.

[62] F. A. Chudnovskii and G. B. Stefanovich, "Metal-insulator phase transition in disordered $\mathrm{VO}_{2}, " J$. Solid State Chem., vol.98, pp.137145, 1992.

[63] M. F. Becker, A. B. Buckman, and R. M. Walser, "Femtosecond laser excitation of the semiconductor-metal phase transition in $\mathrm{VO}_{2}$," Appl. Phys. Lett., vol. 65, p. 1507, 1994.

[64] A. Cavalleri, C. Toth, C. W. Siders, J. A. Squier, F. Raksi, P. Forget, and J. C. Kieffer, "Femtosecond structural dynamics in $\mathrm{VO}_{2}$ during an ultrafast solid-solid phase transition," Phys. Rev. Lett., vol. 87, p. 237401, 2001.

[65] G. B. Stefanovich, A. L. Pergament, A. A. Velichko, and L. A. Stefanovich, "Anodic oxidation of vanadium and properties of vanadium oxide films," J. Phys.: Condensed Matter, vol. 16, pp. 4013-4024, 2004.

[66] A. V. Ilinskiy, O. E. Kvashenkina, and E. B. Shadrin, "Phase transition and correlation effects in vanadium dioxide," Semiconductors, vol. 46, issue 4, pp. 422-429, 2012; A. V. Ilinskiy, O. E. Kvashenkina, and E. B. Shadrin, "Nature of the electronic component of the thermal phase transition in $\mathrm{VO}_{2}$ films," ibidem, issue 9, pp. 1171-1185, 2012.

[67] A. Pergament, "Metal-insulator transition: the Mott criterion and coherence length", J. Phys.: Condensed Matter, vol. 15, no. 19, pp. 3217-3224, 2003

[68] A. L. Pergament, P. P. Boriskov, A. A. Velichko, and N. A. Kuldin, "Switching effect and the metal-insulator transition in electric field," Journal of Physics and Chemistry of Solids, vol. 71, pp. 874-879, 2010.

[69] C. Weber, D. D. O'Regan, N. D. M. Hine, M. C. Payne, G. Kotliar, and P. B. Littlewood, "Vanadium dioxide: A Peierls-Mott insulator stable against disorder," Phys. Rev. Lett., vol. 108, p. 256402, 2012.

[70] J. Laverock, A. R. H. Preston, D. Newby, Jr., K. E. Smith, S. Sallis, L. F. J. Piper, S. Kittiwatanakul, J. W. Lu, S. A. Wolf, M.
Leandersson, and T. Balasubramanian, "Photoemission evidence for crossover from Peierls-like to Mott-like transition in highly strained VO $_{2}$," Phys. Rev., vol. B 86, p. 195124, 2012.

[71] W.-T. Liu, J. Cao, W. Fan, Z. Hao, M. C. Martin, Y. R. Shen, J. Wu, and F. Wang, "Intrinsic Optical Properties of Vanadium Dioxide near the Insulator-Metal Transition," Nano Lett., vol. 11, pp. 466470, 2011

[72] Z. Yang, S. Hart, C. Ko, A. Yacoby, and S. Ramanathan, "Studies on electric triggering of the metal-insulator transition in VO2 thin films between $77 \mathrm{~K}$ and $300 \mathrm{~K}$," Journal of Applied Physics, vol. 110, p. 033725, 2011

[73] T. Driscoll, J. Quinn, M. Di Ventra, D. N. Basov, G. S. Yong-Wook, L. H.-T. Kim, and D. R. Smith, "Current oscillations in vanadium dioxide: Evidence for electrically triggered percolation avalanches," Phys. Rev., vol. B 86, p. 094203, 2012.

[74] M. M. Qazilbash, M. Brehm, B. G. Chae, P.-C. Ho, G. O. Andreev, B. J. Kim, S. J. Yun, A. V. Balatsky, M. B. Maple, F. Keilmann, H. T. Kim, and D. N. Basov, "Mott transition in $\mathrm{VO}_{2}$ revealed by infrared spectroscopy and nano-imaging," Science, vol. 318, no. 5857, pp. 1750-1753, 2007.

[75] V. N. Andreev, V. A. Klimov, and M. E. Kompan, "Influence of hydrogenation on electrical conductivity of vanadium dioxide thin films," Physics of the Solid State, vol. 54, no. 3, pp. 601-606, 2012.

[76] A. V. Ilinskiy, O. E. Kvashenkina, and E. B. Shadrin, "Protonic metallization of the monoclinic phase in $\mathrm{VO}_{2}$ films," Semiconductors, vol. 45, no. 9, pp. 1153-1157, 2011.

[77] J. Wei, H. Ji, W. Guo, A. H. Nevidomskyy, and D. Natelson, "Hydrogen stabilization of metallic vanadium dioxide in singlecrystal nanobeams," Nature Nanotechnology, vol. 7, pp. 357-362, 2012.

[78] J. Nag, R. F. Haglund, Jr., E. A. Payzant, and K. L. More, "Noncongruence of thermally driven structural and electronic transitions in VO2," J. Appl. Phys., vol. 112, p. 103532, 2012.

[79] B. Wu, A. Zimmers, H. Aubin, R. Ghosh, Y. Liu, and R. Lopez, "Electric-field-driven phase transition in vanadium dioxide," Phys Rev., vol. B 84, p. 24141, 2011.

[80] A. L. Pergament, A. A. Velichko, O. Ya. Berezina, E. L. Kazakova, N. A. Kuldin, and D. V. Artyukhin, "Influence of doping on the properties of vanadium oxide gel films," J. Phys.: Condens. Matter, vol. 20, no 42, p. 422204, 2008.

[81] X. Tan, T. Yao, R. Long, Z. Sun, Y. Feng, H. Cheng, X. Yuan, W. Zhang, Q. Liu, C. Wu, Y. Xie, and S. Wei, "Unraveling Metalinsulator Transition Mechanism of $\mathrm{VO}_{2}$ Triggered by Tungsten Doping," Scientific Reports, vol. 2, p.466, 2012.

[82] C. Ko and S. Ramanathan, "Observation of electric field-assisted phase transition in thin film vanadium oxide in a metal-oxidesemiconductor device geometry," Appl. Phys. Lett., vol. 93, p. 252101, 2008

[83] Z. Huang, C. Chen, C. Lv, and S. Chen, "Tungsten-doped vanadium dioxide thin films on borosilicate glass for smart window application," Journal of Alloys and Compounds, vol. 564, pp. 158$161,2013$.

[84] L. Chen, C. Huang, G. Xu, L. Miao, J. Shi, J. Zhou, and X. Xiao, "Synthesis of ThermochromicW-Doped VO2 (M/R) Nanopowders by a Simple Solution-Based Process," Journal of Nanomaterials, vol. 2012, Article ID 491051, 8 pages, 2012.

[85] M. Nazari, C. Chen, A. A. Bernussi, Z. Y. Fan, and M. Holtz, "Effect of free-carrier concentration on the phase transition and vibrational properties of $\mathrm{VO}_{2}$," Appl. Phys. Lett., vol. 99, no. 7, p. 071902, 2011.

[86] M. Hada, K. Okimura, and J. Matsuo, "Photo-induced lattice softening of excited-state VO2," Appl. Phys. Lett., vol. 99, p. 051903, 2011.

[87] G. Seo, B.-J. Kim, Y. W. Lee, and H.-T. Kim, "Photo-assisted bistable switching using Mott transition in two-terminal VO2 device," Appl. Phys. Lett., vol. 100, p. 011908, 2012.

[88] M. Hada, D. Zhang, A. Casandruc, R. J. D. Miller, Y. Hontani, J. Matsuo, R. E. Marvel, and R. F. Haglund, "Hot electron injection driven phase transitions," J. Phys. Rev., vol. B 86, pp.134101, 2012.

[89] S. Wall, L. Foglia, D. Wegkamp, K. Appavoo, J. Nag, R. F. Haglund, Jr., J. Stahler, and M. Wolf, "Tracking the evolution of electronic and structural properties of $\mathrm{VO} 2$ during the ultrafast photoinduced insulator-metal transition," Phys. Rev. B, vol. 87, p. 115126, 2013. 
[90] M. Hada, Y. Hontani, R. E. Marvel, R. F. Haglund, and J. Matsuo, "Ultrafast hot electron induced phase transition in vanadium dioxide," EPJ Web of Conferences, 41, 03005, 2013, http://dx.doi.org/10.1051/epjconf/20134103005

[91] H. Wen, L. Guo, E. Barnes, J. H. Lee, D. A. Walko, R. D. Schaller, J. A. Moyer, R. Misra, Y. Li, E. M. Dufresne, D. G. Schlom, V. Gopalan, and J. W. Freeland, "Structural and electronic recovery pathways of a photoexcited ultrathin VO2 film," Phys. Rev. B, vol. 88, art. no. 165424, 2013.

[92] J. Leroy, A. Crunteanu, A. Bessaudou, F. Cosset, C. Champeaux, and J.-C. Orlianges, "High-speed metal-insulator transition in vanadium dioxide films induced by an electrical pulsed voltage over nano-gap electrodes," Appl. Phys. Lett., vol. 100, p. 213507, 2012.

[93] Y. Zhang and S. Ramanathan, "Analysis of "on" and "off" times for thermally driven $\mathrm{VO} 2$ metal-insulator transition nanoscale switching devices," Solid-State Electronics, vol. 62, pp. 161-164, 2011

[94] A. Kar, N. Shukla, E. Freeman, H. Paik, H. Liu, R. Engel-Herbert, S. S. N. Bhardwaja, D. G. Schlom, and S. Datta, "Intrinsic electronic switching time in ultrathin epitaxial vanadium dioxide thin film," Appl. Phys. Lett., vol. 102, p. 072106, 2013.

[95] J. Wei and D. Natelson, "Nanostructure studies of strongly correlated materials," Nanoscale, vol. 3, pp. 3509-3521, 2011.

[96] E. Morosan, D. Natelson, A. H. Nevidomskyy, and Q. Si, "Strongly Correlated Materials," Advanced Materials, vol. 24, no.36, pp. 48964923, Sep. 2012.

[97] A. Pergament and A. Velichko, "Metal-insulator transition in thin films of vanadium dioxide: The problem of dimensional effects," Thin Solid Films, vol. 518, no. 6, pp. 1760-1762, 2010.

[98] E. Strelcov, A. Tselev, I. Ivanov, J. D. Budai, J. Zhang, J. Z. Tischler, I. Kravchenko, S. V. Kalinin, A. Kolmakov, "Doping-based stabilization of the M2 phase in free-standing VO2 nanostructures at room temperature," Nano Letters, vol. 12 , no. 12, pp. 6198-6205, 2012.

[99] F. Chudnovskiy, S. Luryi, and B. Spivak, "Switching Device Based on a First-Order Metal-Insulator Transition Induced by an External Electric Field," in: Future Trends in Microelectronics: the Nano Millennium. New York: John Wiley and Sons Ltd, 2002.

[100] V. N. Andreev, A. G. Aronov, F. A. Chudnovskii, "Phase transition in electric field in V2O3 and switching effect," Fiz. Tv. Tela [Sov. Phys. Solid State], vol. 12, no. 5, pp. 1557-1559, 1970.

[101] K. A. Valiev, Yu. V. Kopaev, V. G. Mokerov, and A. V. Rakov, "Electron structure and phase transitions in lower vanadium oxides in an electric field," Sov. Phys. JETP, vol. 33, no. 6, pp.1168-1174, Dec. 1971.

[102] V. V. Mokrousov and V. N. Kornetov, "Field effects in vanadium dioxide films," Fiz. Tv. Tela [Sov. Phys. Solid State], vol. 16, no. 10, pp.3106-3107, 1974

[103] G. P. Vasil'ev, I. A. Serbinov, and L. A. Ryabova "Switching in the system VO2-insulator-semiconductor," Pis'ma v ZhTF [Sov. Tech. Phys. Lett], vol.. 3, no. 8, pp.342-344, 1977.

[104] A. A. Kokin, "Metal-insulator phase transition in electric field," Fiz. Tv. Tela [Sov. Phys. Solid State], vol. 17, no.5, pp.1317-1326, 1975.

[105] P. P. Boriskov, A. A. Velichko, A. L. Pergament, G. B. Stefanovich, and D. G. Stefanovich "The Effect of Electric Field on MetalInsulator Phase Transition in Vanadium Dioxide," Technical Physics Letters, vol. 28, no. 5, pp. 406-408, May 2002.

[106] D. Ruzmetov, G. Gopalakrishnan, J. Deng, V. Narayanamurti, and S. Ramanathan, "Electrical triggering of metal-insulator transition in nanoscale vanadium oxide junctions," J. Appl. Phys., vol. 106, p. 083702, 2009.

[107] S. Hormoz and S. Ramanathan, "Limits on vanadium oxide Mott metal-insulator transition field-effect transistors," Solid-State Electronics, vol. 54, pp. 654-659, 2010.

[108] S. Sengupta, K. Wang, K. Liu, A. K. Bhat, S. Dhara, J. Wu, and M. M. Deshmukh, "Field-effect modulation of conductance in VO2 nanobeam transistors with $\mathrm{HfO} 2$ as the gate dielectric," Appl. Phys. Lett., vol. 99, p. 062114, 2011.

[109] N. A. Kuldin, A. A. Velichko, A. L. Pergament, and G. B. Stefanovich, "Numerical simulation of the field effect on metalinsulator transition in vanadium dioxide," in Proc. 2nd International Conference on Physics of Electronic Materials, Kaluga, Russia, 2005, pp. $209-212$.
[110] Y. Zhou and S. Ramanathan, "GaN/VO2 heteroepitaxial p-n junctions: Band offset and minority carrier dynamics," J. Appl. Phys., vol. 113, p. 213703, 2013.

[111] Z. Yang, Y. Zhou, and S. Ramanathan, "Studies on roomtemperature electric-field effect in ionic-liquid gated VO2 threeterminal devices," J. Appl. Phys., vol. 111, p. 014506, 2012); Y. Zhou and S. Ramanathan, "Relaxation dynamics of ionic liquid$\mathrm{VO}_{2}$ interfaces and influence in electric double-layer transistors, " $J$. Appl. Phys., vol. 111, p. 084508, 2012; J. S. Sim, Y. Zhou, and S. Ramanathan, "Suspended sub-50 nm vanadium dioxide membrane transistors: fabrication and ionic liquid gating studies," Nanoscale, vol. 4, pp. 7056-7062, 2012.

[112] M. Nakano, K. Shibuya, D. Okuyama, T. Hatano, S. Ono, M. Kawasaki, Y. Iwasa, and Y. Tokura, "Collective bulk carrier delocalization driven by electrostatic surface charge accumulation," Nature, vol. 487, pp. 459-462, Jul. 2012.

[113] J. Jeong, N. Aetukuri, T. Graf, T. D. Schladt, M. G. Samant, S. S. P. Parkin, "Suppression of Metal-Insulator Transition in $\mathrm{VO}_{2}$ by Electric Field-Induced Oxygen Vacancy Formation," Science, vol. 339, no. 6126, pp. 1402-1405, 2013.

[114] W. W. Li, J. J. Zhu, J. R. Liang, Z. G. Hu, J. Liu, H. D. Chen, and J. H. Chu, "External Electric Field Manipulations on Structural Phase Transition of Vanadium Dioxide Nanoparticles and Its Application in Field Effect Transistor" J. Phys. Chem., vol. 115, p. 23558, 2011.

[115] E. Freeman, A. Kar, N. Shukla, R. Misra, R. Engel-Herbert, D. Schlom, V. Gopalan, K. Rabe, and S. Datta, "Characterization and modeling of metal-insulator transition (MIT) based tunnel junctions," in Proc. 70th Annual Device Research Conference, University Park, TX, 2012 , pp. $243-244$.

[116] Y. Zhou and S. Ramanathan, "Correlated Electron Materials and Field Effect Transistors for Logic: A Review," Critical Reviews in Solid State and Materials Sciences, vol. 38, no. 4, pp. 286-316, 2013.

[117] S. D. Ha and S. Ramanathan, "Adaptive oxide electronics: A review," J. Appl. Phys., vol. 110, p. 071101, 2011.

[118] D. Ruzmetov, G. Gopalakrishnan, C. Ko, V. Narayanamurti, and S. Ramanathan, "Three-terminal field effect devices utilizing thin film vanadium oxide as the channel layer," 2010 [Online]. Available: http://arxiv.org/abs/1006.4373.

[119] W.-K. Hong, J. B. Park, J. Yoon, B.-J. Kim, J. I. Sohn, Y. B. Lee, T.S. Bae, S.-J. Chang, Y. S. Huh, B. Son, E. A. Stach, T. Lee, and M. E. Welland, "Hydrogen-Induced Morphotropic Phase Transformation of Single-Crystalline Vanadium Dioxide Nanobeams," Nano Lett., vol. 14, no. 4, pp. 1822-1828, 2013.

[120] H. T. Kim, K. Y. Kang, D. H. Youn, and B. G. Chae, "Field effect transistor using vanadium dioxide layer as channel material and method of manufacturing the field effect transistor," U.S. Patent 6,933,553, Aug. 2005.

[121] C. Vaju, L. Cario, B. Corraze, E. Janod, V. Dubost, T. Cren, D. Roditchev, D. Braithwaite, and O. Chauvet, "Electric Pulse Induced Resistive Switching, Electronic Phase Separation, and Possible Superconductivity in a Mott insulator," Advanced Materials, vol. 20, pp. 2760-2765, 2008

[122] S. Lee, A. Fursina, J. T. Mayo, C. T. Yavuz, V. L. Colvin, R. G. S. Sofin, I. V. Shvets, and D. Natelson, "Electrically driven phase transition in magnetite nanostructures," Nature Materials, vol. 7, pp. $130-133,2007$

[123] W. Bao, X. Cai, D. Kim, K. Sridhara, and M. S. Fuhrer, "High mobility ambipolar MoS2 field-effect transistors: Substrate and dielectric effects," Appl. Phys. Lett., vol. 102, p. 042104, 2013; G. Fiori, B. N. Szafranek, G. Iannaccone, and D. Neumaier, "Velocity saturation in few-layer MoS2 transistor," Appl. Phys. Lett. vol. 103, p. 233509, Dec. 2013.

[124] M. Kimata, T. Ishihara, A. Ueda, H. Mori, and H. Tajima "Fabrication of a field effect transistor structure using charge-ordered organic materials $\alpha$-(BEDT-TTF $)_{2} \mathrm{I}_{3}$ and $\alpha$ '-(BEDT-TTF $)_{2} \mathrm{IBr}_{2}, "$ Synthetic Metals, vol. 173, pp. 43-45, 2013.

[125] W. L. Lim, E. J. Moon, J. W. Freeland, D. J. Meyers, M. Kareev, J. Chakhalian, and S. Urazhdin, "Field-effect diode based on electroninduced Mott transition in NdNiO3," Appl. Phys. Lett., vol. 101, p. 143111, 2012.

[126] D.M. Newns, T. Doderer, C.C. Tsuei, W.M. Donath, J.A. Misewich, A. Gupta, B.M. Grossman, A. Schrott, B.A. Scott, and P.C. Pattnaik, 
"The Mott Transition Field Effect Transistor: A Nanodevice?" Journal of Electroceramics, vol. 4, no. 2-3, pp. 339-344, 2000.

[127] I. H. Inoue and M. J. Rozenberg, "Taming the Mott Transition for a Novel Mott Transistor," Advanced Functional Materials, vol. 18, no. 16, pp. 2289-2292, 2008.

[128] E. S. Reich, "Metal oxide chips show promise," Nature, vol. 495, p. 17,2013

[129] P.-H. Xiang, S. Asanuma, H. Yamada, H. Sato, I. H. Inoue, H. Akoh, A. Sawa, M. Kawasaki, and Y. Iwasa, "Electrolyte-Gated SmCoO3 Thin-Film Transistors Exhibiting Thickness-Dependent Large Switching Ratio at Room Temperature," Adv. Mater., vol. 25, pp. 2158-2161, 2013.

[130] V. Guiot, L. Cario, E. Janod, B. Corraze, V. Ta Phuoc, M. Rozenberg, P. Stoliar, T. Cren, and D. Roditchev, "Avalanche breakdown in $\mathrm{GaTa}_{4} \mathrm{Se}_{8-x} \mathrm{Te}_{x}$ narrow-gap Mott insulators," Nature Communications, vol. 4, art. no. 1722, 2013.

[131] B. Radisavljevic and A. Kis, "Mobility engineering and a metalinsulator transition in monolayer $\mathrm{MoS}_{2}$," Nature Materials, vol. 12, pp. 815-820, 2013.

[132] S. de Jong, R. Kukreja, C. Trabant, N. Pontius, C. F. Chang, T. Kachel, M. Beye, F. Sorgenfrei, C. H. Back, B. Brauer, W. F. Schlotter, J. J. Turner, O. Krupin, M. Doehler, D. Zhu, M. A. Hossain, A. O. Scherz, D. Fausti, F. Novelli, M. Esposito, W. S. Lee, Y. D. Chuang, D. H. Lu, R. G. Moore, and M. Yi, "Speed limit of the insulator-metal transition in magnetite," Nature Materials, vol. 12, pp. 882-886, 2013.

[133] A. Axelevitch, B. Gorenstein, and G. Golan, "Investigation of the electrical transport mechanism in VOx thin films," Microelectronics Reliability, vol. 51, pp. 2119-2123, 2011.

[134] G. Beydaghyan, V. Basque, and P.V. Ashrit, "High contrast thermochromic switching in vanadium dioxide (VO2) thin films deposited on indium tin oxide substrates," Thin Solid Films, vol. 522 pp. 204-207, 2012

[135] G. Golan and A. Axelevitch, "Electrical transport mechanism in VO2 thin films," in Proc. 27th International Conference on Microelectronics, Nis, Serbia, 2010, pp.141 - 144

[136] M. D. Pickett, G. Medeiros-Ribeiro, and R. S. Williams, "A scalable neuristor built with Mott memristors," Nature Materials, vol. 12, pp. 114-117, Feb. 2013.

[137] A. Zimmers, L. Aigouy, M. Mortier, A. Sharoni, S. Wang, G. West, J. G. Ramirez, and I. K. Schuller, "Role of Thermal Heating on the Voltage Induced Insulator-Metal Transition in VO2," Phys. Rev Lett., vol. 110, p. 056601, 2013.

[138] B. S. Mun, J. Yoon, S.-K. Mo, K. Chen, N. Tamura, C. Dejoie, M. Kunz, Z. Liu, C. Park, K. Moon, and H. Ju, "Role of joule heating effect and bulk-surface phases in voltage-driven metal-insulator transition in VO2 crystal," Appl. Phys. Lett., vol. 103, p. 061902, 2013.

[139] J. H. Park, J. M. Coy, T. S. Kasirga, C. Huang, Z. Fei, S. Hunter, and D. H. Cobden, "Measurement of a solid-state triple point at the metal-insulator transition in VO2," Nature, vol. 500, pp. 431-434, 2013

[140] R. Fors, S. I. Khartsev, and A. M. Grishin, "Giant resistance switching in metal-insulator-manganite junctions: Evidence for Mott transition," Phys. Rev. B, vol. 71, p. 045305, 2005.

[141] S. B. Choi, J. S. Kyoung, H. S. Kim, H. R. Park, D. J. Park, B.-J. Kim, Y. H. Ahn, F. Rotermund, H.-T. Kim, K. J. Ahn, and D. S. Kim, "Nanopattern enabled terahertz all-optical switching on vanadium dioxide thin film," Appl. Phys. Lett., vol. 98, p. 071105, 2011.

[142] M. Liu, H. Y. Hwang, H. Tao, A. C. Strikwerda, K. Fan, G. R. Keiser, A. J. Sternbach, K. G. West, S. Kittiwatanakul, J. Lu, S. A. Wolf, F. G. Omenetto, X. Zhang, K. A. Nelson, and R. D. Averitt, "Terahertz-field-induced insulator-to-metal transition in vanadium dioxide metamaterial," Nature, vol. 487, pp. 345-348, 2012.

[143] A. Crunteanu, J. Leroy, G. Humbert, D. Ferachou, J.-C. Orlianges, C. Champeaux, and P. Blondy, "Tunable Terahertz Metamaterials based on Metal-Insulator Phase Transition of VO2 Layers," Microwave Symposium Digest (MTT), 2012 IEEE MTT-S International, Montreal, QC, Canada, 2012, http://ieeexplore.ieee.org/xpl/articleDetails.jsp?arnumber=6259429.

[144] R. Cabrera, E. Merced, and N. Sepulveda, "A micro-electromechanical memory based on the structural phase transition of VO2," Phys. Status Solidi A, vol. 210, no. 9, pp. 1704-1711, Sep. 2013.
[145] N. Manca, L. Pellegrino, T. Kanki, S. Yamasaki, H. Tanaka, A. S. Siri, and D. Marre, "Programmable Mechanical Resonances in MEMS by Localized Joule Heating of Phase Change Materials," $A d v$ Mater., 2013, doi:10.1002/adma.201302087.

[146] R. Cabrera, E. Merced, N. Davila, F. E. Fernandez, and N. Sepulveda, "A multiple-state micro-mechanical programmable memory," Microelectronic Engineering, vol. 88, pp. 3231-3234, 2011.

[147] H. Kim, Y. Kim, K. S. Kim, H. Y. Jeong, A-R. Jang, S. H. Han, D. H. Yoon, K. S. Suh, H. S. Shin, T. Y. Kim, and W. S. Yang, "Flexible Thermochromic Window Based on Hybridized VO2/Graphene," ACS Nano, vol. 7, no. 7, pp. 5769-5776, 2013.

[148] S.-J. Chang, W.-K. Hong, H. J. Kim, J. B. Lee, J. Yoon, H. C. Ko, and Y.S. Huh, "Probing the photothermally induced phase transitions in single-crystalline vanadium dioxide nanobeams," Nanotechnology, vol. 24, pp. 345701-345708, 2013.

[149] C.G. Granqvist, İ. Bayrak Pehlivan, Y.-X. Ji, S.-Y. Li, and G.A. Niklasson, "Electrochromics and thermochromics for energy efficient fenestration: Functionalities based on nanoparticles of $\operatorname{In}_{2} \mathrm{O}_{3}: \mathrm{Sn}$ and $\mathrm{VO}_{2}$, , Thin Solid Films, Oct. 2013 http://dx.doi.org/10.1016/j.tsf.2013.10.033

[150] P. A. Do, A. Hendaoui, E. Mortazy, M. Chaker, and A. Hache, "Vanadium dioxide spatial light modulator for applications beyond 1200 nm," Optics Communications, vol. 288, pp. 23-26, 2013.

[151] J.D. Budai, A. Tselev, J.Z. Tischler, E. Strelcov, A. Kolmakov, W.J. Liu, A. Gupta, and J. Narayan, "In situ X-ray microdiffraction studies inside individual VO2 microcrystals," Acta Materialia, vol. 61, pp. 2751-2762, 2013.

[152] A. Joushaghani, B. A. Kruger, S. Paradis, D. Alain, J. S. Aitchison, and J. K. S. Poon, "Sub-volt broadband hybrid plasmonic-vanadium dioxide switches," Appl. Phys. Lett., vol. 102, p. 061101, 2013.

[153] L. A. Sweatlock and K. Diest, "Vanadium dioxide based plasmonic modulators," Optics Express, vol. 20, no. 8, pp. 8700-8709, 2012.

[154] A.K. Prasad, S. Amirthapandian, S. Dhara, S. Dash, N. Murali, A.K Tyagi, "Novel single phase vanadium dioxide nanostructured films for methane sensing near room temperature," Sensors and Actuators $B$, vol. 191, pp. 252-256, 2014

[155] S. D. Ha, Y. Zhou, C. J. Fisher, S. Ramanathan, and J. P. Treadway, "Electrical switching dynamics and broadband microwave characteristics of $\mathrm{VO}_{2}$ radio frequency devices," J. Appl. Phys., vol. 113 , p. 184501, 2013

[156] J.-G. Ramírez, R. Schmidt, A. Sharoni, M. E. Gómez, I. K. Schuller, and E. J. Patiño, "Ultra-thin filaments revealed by the dielectric response across the metal-insulator transition in VO2," Appl. Phys. Lett., vol. 102, p. 063110, 2013.

[157] M.-J. Lee, Y. Park, D.-S. Suh, E.-H. Lee, S. Seo, D.-C. Kim, R. Jung, B.-S. Kang, S.-E. Ahn, C. B. Lee, D. H. Seo, Y.-K. Cha, I.-K. Yoo, J.-S. Kim, and B. H. Park, "Two Series Oxide Resistors Applicable to High Speed and High Density Nonvolatile Memory," Adv. Mater., vol. 19, pp. 3919-3923, 2007.

[158] B. H. Banik and H.F. Merkel, "VO2 TES as room temperature THz detectors," in Proc. 17th International Symposium on Space Terahertz Technology, Paris, France, 2006, pp. 18-22.

[159] E. Merced, J. Zhang, X. Tan, and N. Sepulveda, "Robust control of VO2-coated microactuators based on self-sensing feedback," in IEEE/ASME International Conference on Advanced Intelligent Mechatronics: Mechatronics for Human Wellbeing, Novotel Wollongong, Australia, 2013, no. 6584167, pp. 656-661.

[160] C. Batista, R. M Ribeiro, and V. Teixeira, "Synthesis and characterization of VO2-based thermochromic thin films for energyefficient windows," Nanoscale Research Letters, vol. 6, p. 301, 2011

[161] E. Haddad, R. V. Kruzelecky, B. Wong, W. Jamroz, A. Hendaoui, M. Chaker, and P. Poinas, "Large tuneability IR emittance thermal control coating for space applications," in 43rd International Conference on Environmental Systems, 2013. http://arc.aiaa.org/doi/abs/10.2514/6.2013-3436

[162] Y. Kim, S. J. Kelly, A. Morozovska, E. K. Rahani, E. Strelcov, E. Eliseev, S. Jesse, M. D. Biegalski, N. Balke, N. Benedek, D. Strukov, J. Aarts, I. Hwang, S. Oh, J. S. Choi, T. Choi, B. H. Park, V. B. Shenoy, P. Maksymovych, and S. V. Kalinin, "Mechanical Control of Electroresistive Switching," Nano Lett., vol. 13, no. 9, pp. 4068 4074, 2013. 
[163] S.-H. Bae, S. Lee, H. Koo, L. Lin, B. H. Jo, C. Park, and Z. L. Wang, "The Memristive Properties of a Single $\mathrm{VO}_{2}$ Nanowire with Switching Controlled by Self-Heating," Advanced Materials, vol. 25, no. 36, pp. 5098-5103, Sep. 2013.

[164] Y. Sun, X. Xiao, G. Xu, G. Dong, G. Chai, H. Zhang, P. Liu, H. Zhu, and Y. Zhan, "Anisotropic vanadium dioxide sculptured thin films with superior thermochromic properties," Scientific Reports, vol. 3, no. 2756, pp. 1-10, 2013.

[165]H. Yen and L. Long, "Smart or not? A theoretical discussion on the smart regulation capacity of vanadium dioxide glazing," Solar Energy Materials \& Solar Cells, vol. 120, pp. 669-674, 2014.

[166] G. Leahu, R. L. Voti, C. Sibilia, and M. Bertolotti, "Anomalous optical switching and thermal hysteresis during semiconductor-metal phase transition of VO2 films on Si substrate," Applied Physics Letters, vol. 103, art. no. 231114, Dec. 2013.

[167] A. Crunteanu, J. Givernaud, J. Leroy, D. Mardivirin, C. Champeaux, J.-C. Orlianges, A. Catherinot, and P. Blondy, "Voltage- and currentactivated metal-insulator transition in $\mathrm{VO}_{2}$-based electrical switches: a lifetime operation analysis," Sci. Technol. Adv. Mater., vol. 11, p. $065002,2010$.

[168] Y. Zhou, X. Chen, C. Ko, Z. Yang, C. Mouli, and S. Ramanathan, "Voltage-triggered Ultra-fast Metal-insulator Transition in Vanadium Dioxide Switches," IEEE Electron Device Letters, vol. 34, no. 2, pp. 220-222, Feb.2013; http://arxiv.org/ftp/arxiv/papers/1307/1307.3774.pdf

[169] A. L. Pergament, P. A. Boldin, V. V. Kolchigin, and T. G. Stefanovich, "Bistable switching and memory effects in transition metal oxides," Proc. Petrozavodsk State University, vol. 8, no. 121, pp. 118-120, 2011.

[170] V. V. Putrolajnen, A. A. Velichko, G. B. Stefanovich, A. L. Pergament, and N. A. Kuldin, "Method of obtaining of nonvolatile storage element," Rus. Parent 2468471, Apr.2011.

[171] I. P. Radu, K. Martens, S. Mertens, C. Adelmann, X. Shi, H. Tielens, M. Schaekers, G. Pourtoisb, S. Van Elshochtb, S. De Gendtb, M. Heyns, J. A. Kittl, "Vanadium dioxide as a memory material," ECS Transactions, vol. 35, no. 2, pp. 233-243, 2011.

[172] Y. Guo and J. Robertson, "Analysis of metal insulator transitions inVO2 and V2O3 for RRAMs," Microelectronic Engineering, vol. 109, pp. 278-281, 2013.

[173] G. S. Nadkarni and V. S. Shirodkar, "Experiment and theory for switching in $\mathrm{Al} / \mathrm{V}_{2} \mathrm{O}_{5} / \mathrm{Al}$ devices," Thin Solid Films, vol. 105, pp. $115-129,1983$.

[174] M. Kang, I. Kim, S. W. Kim, J-.W. Ryu, and H. Y. Park, "Metalinsulator transition without structural phase transition in $\mathrm{V}_{2} \mathrm{O}_{5}$ film," Applied Physics Letters, vol. 98, p. 131907, 2011.

[175] R. Blum, H. Niehus, C. Hucho R. Fortrie, M. V. GandugliaPirovano, J. Sauer, S. Shaikhutdinov, and H.-J. Freund, "Surface Metal-Insulator Transition on a Vanadium Pentoxide (001) Single Crystal," Physical Review Letters, vol. 99, p. 226103, 2007.

[176] A. Pergament, G. Stefanovich, and V. Andreev, "Comment on "Metal-insulator transition without structural phase transition in V2O5 film" [Appl. Phys. Lett. 98, 131907 (2011)]," Appl. Phys. Lett., vol. 102, p. 176101, 2013.

[177] A. L. Pergament, E. L. Kazakova, and G. B. Stefanovich, "Optical and electrical properties of vanadium pentoxide xerogel films: Modification in electric field and the role of ion transport" J. Phys. D: Appl. Phys., vol. 35, no.17, pp. 2187-2197, 2002.

[178] N. F. Quackenbush, J. W. Tashman, J. A. Mundy, S. Sallis, H. Paik, R. Misra, J. A. Moyer, J.-H. Guo, D. A. Fischer, J. C. Woicik, D. A. Muller, D. G. Schlom, and L. F. J. Piper, "Nature of the Metal Insulator Transition in Ultrathin Epitaxial Vanadium Dioxide," Nano Lett., vol. 13, no. 10, pp. 4857-4861, 2013.

[179] M. Nazari, Y. Zhao, V. Hallum, A. A. Bernussi, Z. Y. Fan, and M. Holtz "Finite size effect on the phase transition of vanadium dioxide," Appl. Phys. Lett., vol. 103, p. 043108, 2013.

[180] T. Fujimoto and K. Awaga, "Electric-double-layer field-effect transistors with ionic liquids," Phys. Chem. Chem. Phys., vol. 15, pp. 8983-9006, 2013.

[181] J. Shi, S. D. Ha, Y. Zhou, F. Schoofs, and S. Ramanathan, "A correlated nickelate synaptic transistor," Nature Communications, vol. 4, Article number: 2676, doi:10.1038/ncomms3676, Jun. 2013. 